\title{
Double-thin-shell Approach to Deriving Total Electron Content From GNSS Signals and Implications for lonospheric Dynamics Near the Magnetic Equator
}

\section{Takashi Maruyama ( $\nabla$ tmaru@nict.go.jp )}

National Institute of Information and Communications Technology https://orcid.org/0000-0003-25561413

Kornyanat Hozumi

NICT

\section{Guanyi Ma}

National Astronomical Observatories Chinese Academy of Sciences

Pornchai Supnithi

KMITL: King Mongkut's Institute of Technology Ladkrabang

\section{Qingtao Wan}

National Astronomical Observatories, Chinese Academy of Sciences

Full paper

Keywords: Global Navigation Satellite System (GNSS), total electron content, double-shell model, arc bias, orthogonal network

Posted Date: December 14th, 2020

DOI: https://doi.org/10.21203/rs.3.rs-125204/v1

License: (9) This work is licensed under a Creative Commons Attribution 4.0 International License. Read Full License 


\title{
RESEARCH
}

\section{Double-thin-shell approach to deriving total electron content from GNSS signals and implications for ionospheric dynamics near the magnetic equator}

\author{
Takashi Maruyama ${ }^{1,2^{*}}$, Kornyanat Hozumi ${ }^{2}$, Guanyi Ma ${ }^{1}$, Pornchai Supnithi ${ }^{3}$ and Qingtao Wan ${ }^{1}$
}

${ }^{*}$ Correspondence:

tmaru@nict.go.jp

${ }^{2}$ National Institute of Information and Communications Technology,

2-1 Nukuikita 4-chome, 184-8795

Tokyo, Japan

Full list of author information is available at the end of the article

\begin{abstract}
A new technique was developed to estimate the ionospheric total electron content (TEC) from Global Navigation Satellite System (GNSS) satellite signals. The vertically distributed electron density was parameterized by two thin-shell layers (double-shell approach). The spatiotemporal variation of TEC (strictly speaking, partial electron content) associated with each shell was approximated by the functional fitting of spherical surface harmonics. The major improvements over the conventional single-shell approach were as follows: (1) the precise estimation of TEC was achieved; (2) the estimated TEC was less dependent on the choice of shell height; and (3) the equatorial anomaly was captured more correctly. Furthermore, higher and lower shells exhibited a different pattern of local time vs latitude variation, providing information on the ionosphere-thermosphere dynamics.
\end{abstract}

Keywords: Global Navigation Satellite System (GNSS); total electron content; double-shell model; arc bias; orthogonal network

\section{Introduction}

Ionosondes have been used as a standard technique of measuring ionospheric characteristics for more than seven decades. Over the last two decades, beacon signals transmitted from the global navigation satellite system (GNSS) satellites have been widely utilized for ionosphere studies. These two methods detect different parameters of the ionosphere. The primary parameter obtained from ionosondes is the electron density $(N m F 2)$ at the peak height, and the primary parameter measured by the satellite beacon signals is total electron content (TEC), in which electron density is integrated along the satellite-to-receiver path. Thus, they are often used complementarily for ionosphere and upper-atmosphere studies. However, the technique of deriving TEC from beacon signals is less established than that of $N m F 2$ measurement, and different approaches yield different TECs.

TEC is calculated from measurements of the differential propagation delay between two frequencies that are transmitted from GNSS satellites and propagated through the dispersive medium. A basic problem is that the signal delays in the electronics of satellites and receivers are different for the two frequencies, which is often referred to as an inter-frequency instrumental bias. Various algorithms have been proposed to remove instrumental biases using a single receiver (Lanyi and Roth 
1988; Arikan et al. 2008; Zhang et al. 2009; Choi et al. 2013; Prasad et al. 2016), a regional or local network of receivers (Otsuka et al. 2002; Ma and Maruyama 2003; Ma et al. 2005; Choi et al. 2011), and a global receiver network (Mannucci et al. 1998; Hernández-Pajares et al. 1999). Regarding the global receiver network, several organizations provide TEC in the form of global ionosphere maps (GIMs) along with instrumental biases (see Hernández-Pajares et al. 2009). However, different approaches based on different assumptions and procedures yield different TECs and biases regardless of whether a single receiver or a network of receivers is used (Brunini et al. 2005; Hernández-Pajares et al. 2009).

The primary observable is the differential group delay $(\Delta \tau)$ including instrumental biases. The ionospheric delay (slant TEC) is a function of the inclination of the ray path, while the instrumental biases are independent of the inclination. Because of this feature, two variables can be separated. In the separation process, the slant TEC must be converted to the vertical value or vertical TEC at a reference point on the ray path by multiplying by the cosine of the local zenith angle $(\chi)$. For this purpose, the ionosphere is expressed by a thin shell and $\chi$ is represented by the value at the point where the ray path intercepts the shell (ionosphere piece point (IPP)). Most techniques use the thin-shell approximation with a constant shell height. However, there are some problems with the thin-shell approximation: (1) the ionospheric peak height and vertical electron density profile largely vary with the location and time; (2) a different shell height connects the observed slant TEC with a different geographical location of the IPP; and (3) the slant TECs differ at the same IPP but different ray path directions under horizontal gradient of TEC. Thus, there is no theoretical criterion for choosing the shell height. A detailed discussion of these problems is given by Hernández-Pajares et al. (1999).

The ionospheric group delay measured by the modulation code gives an absolute value of slant TEC except that instrumental biases are included. However, the data are subject to noises and the multipath effect. On the other hand, the carrier phase advance gives the same ionospheric effect as the group delay but with a different sign, and with less noises and a reduced multipath problem. Because the origin of each series of continuous phase data is unknown (phase ambiguity), the absolute value is obtained by least-squares fitting to the code data, which is called code-phase leveling and commonly used to derive TEC precisely. Code-phase data leveling, however, is not free of noises or the multipath effect on the code data (Hernández-Pajares et al. 1999).

In this paper, to resolve the problems inherent in the conventional techniques, 1. the three-dimensional ionosphere was parameterized by two thin shells and the spatiotemporal distribution of TEC (more strictly, partial electron content) associated with each shell was modeled by spherical harmonics; and 2 . the phase data were directly used without leveling, where the arc biases containing instrumental biases and phase ambiguity are defined. The algorithm is described in section 1 . The technique is first validated for simulated slant TEC data in section 2. The application to an actual dataset follows in section 3 . The discussion and interpretation of the results are given in section 4 . Section 5 shows the advantage of the new technique. 
${ }_{93} \quad \phi_{i}=\frac{T}{86400} 2 \pi+\zeta_{i}$

\section{Algorithm}

\subsection{Conversion of measurements} follows:

$\theta_{i}=\frac{\pi}{2}-\operatorname{modip}\left(\lambda_{i}, \zeta_{i}\right)$

The technique was developed to apply to local receiver networks along a meridian, although it is applicable in single-receiver cases. To adopt the surface harmonics fitting approach, data obtained in a whole day were prepared and the local time of IPP or the angular local time, which covers the range $0-2 \pi$, was used as the longitudinal argument $(\phi)$ of the surface harmonic function. The colatitude argument $(\theta)$ was the co-angle of the modified dip latitude (modip), which is suitable for the analysis of the equatorial and low-latitude ionosphere. The differential carrier phase advance $(-\Delta \tau)$ between the two frequencies of GNSS satellites was calculated from RINEX data files. The consecutive phase data set with $30 \mathrm{~s}$ increments for a given receiver and satellite combination was numbered (arc number).

The ionosphere effect on radio wave propagation was assumed to be the summation of the contributions in two thin-shell layers located at two constant heights (double-shell approximation). The azimuth $(\gamma)$ and elevation $(\eta)$ of the satellite were converted to the IPP parameters as shown in Figure 1. The latitude $\left(\lambda_{i}\right)$ and longitude $\left(\zeta_{i}\right)$ of the IPP in each shell and the local satellite zenith angle $\left(\chi_{i}\right)$ ( $i=L o$ and $H i$ for the lower and higher shells, respectively) were calculated using the same equation set as that used in the single-thin-shell approximation.

$$
\begin{aligned}
\lambda_{i} & =\sin ^{-1}\left(\sin \lambda_{R} \cos \alpha_{i}+\cos \lambda_{R} \sin \alpha_{i} \cos \gamma\right) \\
\zeta_{i} & =\zeta_{R}+\sin ^{-1}\left(\frac{\sin \alpha_{i} \sin \gamma}{\cos \lambda_{i}}\right) \\
\chi_{i} & =\sin ^{-1}\left(\frac{R_{E}}{R_{E}+h_{s i}} \cos \eta\right) \\
{ }_{86} & =\frac{\pi}{2}-\eta-\chi_{i}
\end{aligned}
$$

Here, $\lambda_{R}$ and $\zeta_{R}$ are the latitude and longitude of the receiver, respectively, $R_{E}$ is the earth's radius, and $h_{s i}$ is the shell height. The coordinates of the IPP are further converted to two arguments, colatitude $\left(\theta_{i}\right)$ and angular local time $\left(\phi_{i}\right)$, as 


\subsection{Surface harmonics fitting}

Ionosphere pierce points in the thin shell are scattered over a wide range of latitude and longitude (and thus local time) around the ground receiver, and they move with time. Thus, vertical TECs associated with each shell, $T E C_{V L o}^{*}$ and $T E C_{V H i}^{*}$, at different IPPs and times are represented by the surface harmonics of the local time in terms of angle $\left(\phi_{i}\right)$ and colatitude $\left(\theta_{i}\right)$ as follows:

2

$$
\begin{aligned}
T E C_{V L o}^{*} & =\sum_{m=0}^{M} \sum_{n=m}^{N}\left(A_{n m} \cos m \phi_{L o}+B_{n m} \sin m \phi_{L o}\right) P_{n}^{m}\left(\cos \theta_{L o}\right) \\
T E C_{V H i}^{*} & =\sum_{m=0}^{M} \sum_{n=m}^{N}\left(\tilde{A}_{n m} \cos m \phi_{H i}+\tilde{B}_{n m} \sin m \phi_{H i}\right) P_{n}^{m}\left(\cos \theta_{H i}\right)
\end{aligned}
$$

where $P_{n}^{m}\left(\cos \theta_{i}\right)$ is the associated Legendre polynomial with degree $n$ and order $m$. The coefficients $A_{n m}, B_{n m}, \tilde{A}_{n m}$, and $\tilde{B}_{n m}$ are unknown parameters, which should be determined from the observations. The maximum degree and order were chosen as $N=9$ and $M=7$, respectively, in all runs in this paper. These values were a compromise between the reproducibility of small-scale spatiotemporal variation of TEC and credibility of the results, which depends on the number of IPPs and noises. The available observations are the slant TECs, $T E C_{s l}^{o b s}$, which are calculated from the differential phase advance. Here, the basic problem is

$$
T E C_{s l}=T E C_{s l}^{o b s}+b_{a r c}
$$

The arc bias $\left(b_{a r c}\right)$, including the instrumental biases and the ambiguity of the phase origin, is considered to be a constant during an arc of continuous phase data.

\subsection{Orthogonal network}

The surface harmonics fitting (or the determination of coefficients $A_{n m}, B_{n m}, \tilde{A}_{n m}$, and $\tilde{B}_{n m}$ ) and the estimation of $b_{\text {arc }}$ were simultaneously carried out by an orthogonal network (Yang and Tseng 1996) with an additional network for bias estimation (Ma et al. 2005) as shown in Figure 2. The IPP coordinates $\theta_{i}$ and $\phi_{i}$ are fed to the nodes of the orthogonal function layer (surface harmonics) and each node calculates $\cos m \phi_{i} P_{n}^{m}\left(\cos \theta_{i}\right)$ or $\sin m \phi_{i} P_{n}^{m}\left(\cos \theta_{i}\right)$. For convenience of explanation, (7) and (8) are rewritten as

$$
\begin{aligned}
& T E C_{V L o}^{*}=x_{L o}=\sum_{j=1}^{K} w_{j} O_{j} \\
& T E C_{V H i}^{*}=x_{H i}=\sum_{j=1}^{K} u_{j} O_{K+j}
\end{aligned}
$$

$$
\text { where } K=M(2 N-M+1)+N+1 \text {. In (10) and (11), } w_{j} \text { and } u_{j} \text { correspond to }
$$
$A_{n m}, B_{n m}, \tilde{A}_{n m}$, and $\tilde{B}_{n m}$.

The diurnal variation of TEC is roughly described by the combination of a half cosine part corresponding to daytime and a flat part corresponding to nighttime 
150

151

$$
\begin{aligned}
\Delta b_{a r c} & =-\frac{\partial E}{\partial b_{a r c}} \\
& =-\left[\frac{\hat{\sigma}\left(x_{L o}\right)}{\cos \chi_{L o}}+\frac{\hat{\sigma}\left(x_{H i}\right)}{\cos \chi_{H i}}-\left(T E C_{s l}^{o b s}-b_{a r c}\right)\right]
\end{aligned}
$$
slant TEC below.

$$
E=\frac{1}{2}\left[\frac{\hat{\sigma}\left(x_{L o}\right)}{\cos \chi_{L o}}+\frac{\hat{\sigma}\left(x_{H i}\right)}{\cos \chi_{H i}}-\left(T E C_{s l}^{o b s}-b_{a r c}\right)\right]^{2}
$$
below and the update was repeated until the residual error converged.

$$
\begin{aligned}
\Delta w_{j}= & -\frac{\partial E}{\partial w_{j}} \\
= & -\left[\frac{\hat{\sigma}\left(x_{L o}\right)}{\cos \chi_{L o}}+\frac{\hat{\sigma}\left(x_{H i}\right)}{\cos \chi_{H i}}-\left(T E C_{s l}^{o b s}-b_{a r c}\right)\right] \\
& \times \frac{1}{\cos \chi_{L o}} \frac{\partial \hat{\sigma}\left(x_{L o}\right)}{\partial x_{L o}} O_{j}
\end{aligned}
$$

$$
\begin{aligned}
\Delta u_{j}= & -\frac{\partial E}{\partial u_{j}} \\
= & -\left[\frac{\hat{\sigma}\left(x_{L o}\right)}{\cos \chi_{L o}}+\frac{\hat{\sigma}\left(x_{H i}\right)}{\cos \chi_{H i}}-\left(T E C_{s l}^{o b s}-b_{a r c}\right)\right] \\
& \times \frac{1}{\cos \chi_{H i}} \frac{\partial \hat{\sigma}\left(x_{H i}\right)}{\partial x_{H i}} O_{K+j}
\end{aligned}
$$

where $i=L o$ and $H i$ for the lower and higher shells, respectively. The graph of the function $\hat{\sigma}$ is shown in Figure 3. When $x$ is larger than a few TECu, TEC $C_{V i} \simeq T E C_{V i}^{*}$ and the activation function has no effect on the output. When $x$ is negative, the functional value remains positive and reaches zero at a large negative $x$ value. In the double-shell parameterization of the ionosphere, only the sum of the contributions from the two shells is compared with the observations. Therefore, even if the sum is positive, TEC associated with one of the two shells can be negative. The activation function in the output layer avoids an unrealistic local minimum in which one of the two shells becomes negative. Thus, (10) - (13) are used instead of (7) and (8).

Functional fitting was performed to minimize the squared residual error of the

The weights and biases were updated by the gradient descent algorithm as described 
The weights and biases at the step $s$ are updated to those at the step $s+1$ with the learning rates $\mu$ and $\tilde{\mu}$ as

$$
\begin{aligned}
& w_{j}^{s+1}=w_{j}^{s}+\mu \Delta w_{j} \\
& u_{j}^{s+1}=u_{j}^{s}+\mu \Delta w_{j} \\
& b_{\text {arc }}^{s+1}=b_{\text {arc }}^{s}+\tilde{\mu} \Delta b_{\text {arc }}
\end{aligned}
$$

In the update process, it is important to choose appropriate learning rates to ensure stability. For a given $n$, the amplitude of $P_{n}^{m}$ varies greatly with $m$ because it is not normalized. Instead, $\mu$ was scaled by the mean of the square of the spherical surface harmonics over the surface $\left(S_{n m}\right)$.

$$
\begin{aligned}
& S_{n m}=\frac{(n+m) !}{2(2 n+1)(n-m) !} \\
& \mu=\mu_{0} / S_{n m}
\end{aligned}
$$

The update was carried out in two stages. First, weights and biases were updated after each pattern was presented in a randomized order (pattern-mode learning) (Haykin 1994). The residual error decreased rapidly in the first $\sim 100$ repetitions and then gradually. The error had almost converged by approximately 1000 to 1500 repetitions. However, the error fluctuated with the update step depending on the order of pattern presentation, which was shuffled at each epoch of repetition. Further updates were carried out by batch-mode learning, i.e., averages of $\Delta w_{j}, \Delta u_{j}$, and $\Delta b_{\text {arc }}$ calculated for all patterns were used for updates (Haykin 1994). In all runs in this paper, $\mu_{0}$ was 0.00001 and 0.001 in the pattern- and batch-mode learning, respectively, with $\tilde{\mu}=K \mu$.

A drawback of the harmonics fitting applied here is the discontinuity of TECs at the start and end of the one-day period, which were forced to be the same in the fitting with the periodic function, while the ionospheric daily variations are not exactly periodic. To mitigate this problem, the daily dataset was chosen to start immediately before sunrise and to end at the same time on the next day, around which TEC generally takes a minimum and the day-to-day variability is the smallest.

\section{Validation by IRI simulation}

When evaluating the vertical TEC estimated from slant TEC measurements, the fitting error is often used as a measure of the correctness of the results. However, it is not a suitable parameter when both biases and TECs are simultaneously determined, because the bias and TEC errors compensate for each other. Thus, to validate the developed technique, we needed to evaluate the bias and vertical TEC errors separately. However, this is not easy from actual observations. The validation was first performed for simulated slant TEC data, and the application to actual observations will be discussed in the next section. To demonstrate the advantage of the double-shell approach, the same evaluation was conducted for the single-shell 
Table 1 GNSS receivers used in this study

\begin{tabular}{llcc}
\hline Code & Explanation & Latitude $\left({ }^{\circ} \mathrm{N}\right)$ & Longitude $\left({ }^{\circ} \mathrm{E}\right)$ \\
\hline CMU0 & Chiangmai & 18.76 & 98.93 \\
UDON & Udonthani & 17.41 & 102.78 \\
NKSW & Nakornsawan & 15.69 & 100.11 \\
KMI0 & Bangkok & 13.73 & 100.78 \\
PJRK & Prachuabkirikhan & 11.81 & 99.80 \\
SRTN & Suratthani & 9.13 & 99.33 \\
SOKA & Songkha & 7.21 & 100.60 \\
KTB2 & Kototabang & -0.20 & 100.32 \\
\hline
\end{tabular}

approach using a reduced version of the double-shell model. The single-shell model was realized by simply removing the higher-shell part in the diagram shown in Figure 2 .

The International Reference Ionosphere model (IRI2016) (Bilitza et al. 2017) was used to calculate slant TECs. For a realistic simulation, the slant paths were adopted from the actual observation geometry. The observations were carried out using eight receivers composed of a network aligned along the $100^{\circ} \mathrm{E}$ meridian near the magnetic equator in Southeast Asia, as summarized in Table 1. GPS data were selected for the period from 22 UT, 16 March 2014 to 22 UT, 17 March 2014, starting and ending immediately before sunrise. The total number of arcs was 564. The IPPs at 300 and $600 \mathrm{~km}$ are shown in Figure 4. The electron density was integrated along the ray path up to a height of $2000 \mathrm{~km}$, which is the upper boundary of the IRI model. Arc biases were randomly generated within $\pm 25 \mathrm{TECu}$ and added to the calculated slant TECs. Vertical TECs were directly calculated for use as a reference for evaluating the estimated vertical TECs. A map of the directly calculated TEC (IRI-direct TEC) as a function of local time and latitude is shown in Figure 5a. The dashdotted line indicates the magnetic equator. Along with the diurnal variation, the characteristics of the equatorial anomaly with a trough over the magnetic equator and crests away from the magnetic equator are depicted.

\subsection{Single-shell results}

In single-shell approaches, the shell height where IPPs are located is usually fixed slightly above the $F$-peak. However, there is no solid basis for this height and, moreover, the height of the $F$-peak varies greatly, especially in the equatorial region. In the case of the IRI model, the peak height varied from 280 to $455 \mathrm{~km}$ under the current conditions in the area shown in Figure 4.

For the above reason, three different shell heights, 350, 450, and $550 \mathrm{~km}$, were examined. The reconstructed maps of TEC as a function of local time and latitude are shown in Figures $5 \mathrm{~b}-5 \mathrm{~d}$. Figures $5 \mathrm{e}-5 \mathrm{~g}$ show the reconstruction errors (difference between the reconstructed and IRI-direct TEC maps). Two types of systematic error were revealed: (1) TEC was underestimated when the shell height was small, and TEC increased with increasing shell height; and (2) the ratio of TEC at the equatorial anomaly crests to that at the trough was small irrespective of the shell height. In other words, the equatorial ionization anomaly was flattened by the reconstruction.

In general the vertical TEC is derived from the slant TEC to minimize the residual error composed of both biases and TECs. Because two parameters are simultaneously solved, the bias and TEC errors compensate for each other. Figures $6 \mathrm{a}-6 \mathrm{c}$ 
show the bias errors for the three shell heights. The horizontal axis shows the randomly generated arc biases and the vertical axis shows the estimated arc biases. When the shell height was chosen as $350 \mathrm{~km}$, the biases were overestimated and the TECs were underestimated as shown in Figure 5e. In contrast, the biases were underestimated for the shell height of $550 \mathrm{~km}$ and the TECs were overestimated as shown in Figure 5g. Thus, the bias and overall TEC errors compensated for each other. When the shell height was taken as $450 \mathrm{~km}$, the bias error was widely scattered around the diagonal line corresponding to the flattening of the equatorial anomaly. Figure 7 shows the residual error of fitting, the root mean square error (RMSE) of the arc bias, and the RMSE of the slant TEC for different shell heights. The shell height giving the minimum fitting error was $\sim 390 \mathrm{~km}$, but a suitable shell height for the TEC derivation was $\sim 430 \mathrm{~km}$.

\subsection{Double-shell results}

In the double-shell approach, it is rational to choose one shell below the $F$ peak and the other shell above the $F$ peak. In addition, the TECs associated with each shell should not differ greatly. Thus, the selection of heights is limited compared with that in the case of the single-shell approach. The three cases examined were the combinations of $250 / 600,300 / 600$, and $300 / 700 \mathrm{~km}$ for the lower/higher shells. The results are shown in Figure 8. The left panels show the vertical TEC maps for each shell height combination and the right panels show the errors. For comparison, the same color code as in Figures 5(e)-5(g) is used in the error maps. At a glance, it can be seen that the three vertical TEC maps are almost identical, and the errors were less than $\pm 1 \mathrm{TECu}$ irrespective of the shell heights. The equatorial anomaly was correctly reproduced.

Arc bias errors are shown in the lower three panels of Figure 6 for the above three combinations of shell heights. The biases were also correctly estimated for all combinations and the scatter was much smaller than that for the single-shell model in the upper panels.

\section{Application to actual observations}

The advantage of the double-shell approach has been validated by simulation using the data set generated by the IRI model. The technique was then applied to the actually observed slant TEC. The data set has already been described, for which the ray path geometry was used to simulate the slant TEC by the IRI model. Differently from the order in the previous section, the double-shell results are first described, then a possible error in the single-shell results is discussed.

\subsection{Double-shell results}

If the two shell heights are suitably chosen, the TEC associated with the lower shell (referred to as the lower-shell TEC or ls-TEC hereafter) decreases and that associated with the higher shell (referred to as the higher-shell TEC or hs-TEC hereafter) increases when the ionosphere is raised by the $\mathbf{E} \times \mathbf{B}$ drift. On the other hand, downward plasma flux along the magnetic field line due to the gravitational force and neutral drag may decrease hs-TEC and increase ls-TEC. Thus, the TEC maps for the lower and higher shells should behave complementarily for several features. 
We postulate that ls-TEC and hs-TEC should be similar amplitudes, and the two combinations of shell heights were taken as $300 / 600$ and $280 / 700 \mathrm{~km}$ for $h_{s L o} / h_{s H i}$. Figures 9a-9c show vertical TEC, ls-TEC, and hs-TEC for shell heights of 300/600 $\mathrm{km}$. Figures $9 \mathrm{~d}-9 \mathrm{f}$ show the same results for shell heights of $280 / 700 \mathrm{~km}$. The two results of vertical TEC were almost identical and the maxima of ls-TEC and hs-TEC were similar for both combinations.

Notable features of the TEC maps in Figures 9a and 9d were the high equatorial anomaly crests around 12-16 LT and the deep equatorial hole-like trough centered at $21 \mathrm{LT}$. The patterns of the ls-TEC and hs-TEC maps were different from each other and also from the TEC maps for either height combination. The equatorial anomaly was prominent in ls-TEC, but hs-TEC exhibited only small crests at around $13 \mathrm{LT}$. The evening equatorial hole was observed only in ls-TEC and was wider than that in the TEC map. In the development stage of the hole in ls-TEC, hs-TEC reached a secondary peak at 18.5 LT. Such distinctive features of ls-TEC and hs-TEC are discussed later from the standpoint of ionospheric dynamics.

\subsection{Single-shell results}

In the single-shell runs, the same shell heights as those in the IRI simulation were chosen. The TEC maps are shown in the left panels of Figure 10 for shell heights of 350,450 , and $550 \mathrm{~km}$. Although the correct values of vertical TEC are unknown, unlike in the IRI simulations, the double-shell results are presumed to be close to the correct TEC in analogy with the IRI simulations. Then, the error of the single-shell results was evaluated with reference to the double-shell results. The right panels of Figure 10 are $\triangle \mathrm{TEC}$, the difference between the left panels and the double-shell TEC with shell heights of $300 / 600 \mathrm{~km}$. When the shell height was taken as 350 km, $\triangle$ TEC was negative everywhere, as shown in Figure 10d, and the largest errors appeared near the equatorial anomaly crests and in a wide range of latitudes in the evening hours. When the shell height was $450 \mathrm{~km}$, the daytime equatorial and nighttime TECs were close to the reference. However, $\triangle$ TEC remained negative near the anomaly crests with the largest error around the evening hours, as shown in Figure 10e. When the shell height was increased further to $550 \mathrm{~km}$, TEC increased further. At around 20 LT, the error was still negative near the anomaly crests and positive around the equator.

Although the error of the single-shell model applied to the actual data was generally similar to that in the IRI simulations, it was larger and more complex than in the simulations. The error in Figure 10e with a shell height of $450 \mathrm{~km}$ ranged from -8 to $4 \mathrm{TECu}$, while the error in Figure $5 \mathrm{f}$ for the same shell height ranged from -4 to $2 \mathrm{TECu}$. The largest error in the actual observations was concentrated around the hour of the evening enhancement in the right panels of Figure 10. The actual ionosphere height on an individual day will be more variable with the time and space than the climatological IRI prediction because the electric field and neutral wind are subject to a large day-to-day variability.

\section{Discussion}

In the previous sections, it was shown that the double-shell model was more suitable for capturing the vertical TEC variations correctly than the single-shell model, especially in the equatorial anomaly region. Furthermore, ls-TEC and hs-TECs varied in 
a different way with the local time and latitude, which can be ascribed to the ionospheric dynamics. In other words, the different behaviors of TECs associated with each shell are expected to provide information on the ionospheric dynamics such as the $\mathbf{E} \times \mathbf{B}$ drift and the field-aligned diffusion induced by the gravitational force and neutral drag. From this perspective, the results in Figure 9 for the individual shells were interpreted.

\subsection{Complementary features between two shells}

The diurnal variations of vertical TEC, ls-TEC, and hs-TEC at the magnetic equator $\left(7.6^{\circ} \mathrm{N}\right.$ geographic latitude as shown by the dash-dotted line in Figures $\left.9 \mathrm{a}-9 \mathrm{c}\right)$ and away from the equator $\left(12^{\circ} \mathrm{N}\right.$ magnetic latitude, $15.9^{\circ} \mathrm{N}$ geographic latitude as shown by the red dashed line in Figures 9a-9c) are shown in Figure 11 for the shell height combination of $300 / 600 \mathrm{~km}$.

TEC first started to increase in the lower shell at around sunrise and then in the higher shell with a delay of 1-2 hours. The increase in ls-TEC is an immediate response to the start of photoionization at 150-200 km. However, the increase in hs-TEC is due to the refilling of the topside ionosphere by the upward plasma flux along the magnetic field line, which caused the delayed response. The upward $\mathbf{E} \times \mathbf{B}$ drift might also have contributed to the increase in hs-TEC.

A notable change occurred at the off-equator latitude when ls-TEC reached a peak slightly before $11 \mathrm{LT}$. After that time, ls-TEC and hs-TEC varied complementarily or out of phase with each other for two cycles until 22 LT. A similar variation started 1.5 hours earlier at the magnetic equator and continued until $23 \mathrm{LT}$ with the exception of the period of 18.5-20 LT, during which ls-TEC markedly decreased. These complicated variations were the manifestation of the ionospheric dynamics, and the difference in TEC between the two shells was further interpreted by focusing on events from hour to hour.

\subsection{Development of equatorial anomaly}

The equatorial anomaly started developing at around 10 LT as shown in Figure 9a. At the same time, ls-TEC at the equator started decreasing and formed a bite-out around noon in Figures $9 \mathrm{~b}$ and 11b. On the other hand, hs-TEC at the equator continued to increase and reached a peak slightly after the lower-shell bite-out in Figure $9 \mathrm{c}$ and $11 \mathrm{~b}$. Figure 12a shows the latitudinal variation of the TECs at 11 LT (dashed lines) and 12 LT (solid lines). During the intervening hour, hs-TEC increased and ls-TEC decreased at all latitudes. The chain of TEC variations is ascribed to the intense upward $\mathbf{E} \times \mathbf{B}$ drift, consistent with the development of the equatorial anomaly.

After 12.5 LT, ls-TEC started to increase and hs-TEC started to decrease in Figure 11. This is interpreted as a weakening of the eastward electric field and the dominant field-aligned plasma diffusion toward lower latitudes in the topside ionosphere. While the downward plasma flux maintained the large crest TEC in Figure $9 \mathrm{a}$, the crest latitude receded toward the equator as a consequence of the weakening of the electric field. The increase in ls-TEC at the trough in Figure 9b reflects the weakened fountain effect and the dominant photoionization. 


\subsection{Evening enhancement}

A transition occurred at $17 \mathrm{LT}$ when hs-TEC was increasing again and ls-TEC was decreasing in Figure 11. The secondary peak of hs-TEC appeared at 18-19 LT in Figure 9c, which was earlier and more intense at latitudes closer to the magnetic equator. Slightly delayed from the hs-TEC peak, a large depression appeared in ls-TEC in Figure 9b. This is clearly due to the effect of the evening enhancement of the upward $\mathbf{E} \times \mathbf{B}$ drift (Fejer et al. 2008). The latitudinal variations of the TECs at 17 and 18 LT are shown in Figure 12b. Unlike in Figure 12a, the decrease in ls-TEC was significant and the two-shell TEC never increased, which is due to the absence of further photoionization. The center of the equatorial depression or the hole of ls-TEC was at $20 \mathrm{LT}$ in Figures $9 \mathrm{~b}$ and $11 \mathrm{~b}$, approximately one hour earlier than that of the center of the two-shell TEC depression in Figures 9a and 11b. The reversal of $\mathbf{E} \times \mathbf{B}$ drift was estimated to occur at 20 LT. Figures $12 \mathrm{c}$ and $12 \mathrm{~d}$ exhibit the reversal of the magnitudes of ls-TEC and hs-TEC in intervening hours caused by the large downward $\mathbf{E} \times \mathbf{B}$ drift. Associated with the reversal of the electric field, the equatorial anomaly crests receded toward the equator after 20 LT in Figure 9a.

\subsection{Hemispheric asymmetry}

Another interesting feature observed in Figure 9 is the north-south asymmetry of TEC. The asymmetry was prominent at the anomaly crests in the ls-TEC maps of Figures $9 \mathrm{~b}$ and $9 \mathrm{e}$, in which the northern anomaly crest was higher than the southern anomaly crest during daytime and in the evening hours. The asymmetry was reversed at $21 \mathrm{LT}$ and continued until midnight.

In the meridional plots, ls-TEC was less than hs-TEC near the magnetic equator as shown in Figures 12a-12c. The magnitudes of ls-TEC and hs-TEC reversed towards off-equatorial latitudes in the northern hemisphere. In Figure 12a, during the development phase of the equatorial anomaly, hs-TEC increased and ls-TEC decreased at all latitudes in the hour from 11 to $12 \mathrm{LT}$, which has already been ascribed to the upward movement of the $F$ peak due to the vertical $\mathbf{E} \times \mathbf{B}$ drift. The increase in hs-TEC and the decrease in ls-TEC were more significant at offequatorial latitudes in the southern hemisphere than in the northern hemisphere. In other words, the $F$ peak in the southern hemisphere rose more rapidly than that in the northern hemisphere, which is ascribed to the effect of upward (northward) field-aligned plasma flow.

In Figure 12b, the equatorial anomaly almost disappeared in the two-shell TEC. The curve of hs-TEC was convex upwards and that of ls-TEC was vertex down, shaping a dome centered at the magnetic equator. In the northern hemisphere, the ratios of the TEC at off-equatorial latitudes to that at the equator for the higher and lower shells were large in the northern hemisphere. Plasma pileup in the lower shell and a depression in the higher shell occurred in the northern hemisphere, which are ascribed to the downward (northward) field-aligned plasma flows.

The lower shell became almost empty around the equator, and the equatorial anomaly developed greatly in the two-shell TEC in Figure 12c. The plasma pileup persisted in the lower shell in the northern hemisphere, but hs-TEC was symmetric about the equator. The equatorward neutral wind was dominant in the northern hemisphere. Two hours later, the relative magnitudes of ls-TEC and hs-TECs was 
reversed, as shown in Figure 12d, indicating the reversal of the $\mathbf{E} \times \mathbf{B}$ drift. The large reduction in TEC in the northern hemisphere is striking. For comparison, the two-shell TEC at 20 LT is reproduced in Figure 12d by the purple dotted line. At the same time, the north-south asymmetry of ls-TEC was reversed. hs-TEC also exhibited a greater reduction in the northern hemisphere, which suggests that the reduction in TEC was due to chemical recombination under a large downward $\mathbf{E} \times \mathbf{B}$ drift. The reduction in TEC in the southern hemisphere was not significant in spite of the large downward $\mathbf{E} \times \mathbf{B}$ drift. The north-south asymmetry of TEC reduction is ascribed to the ion drag of northward neutral wind, such that the downward field-aligned plasma flow enhanced the descent of the $F$ layer due to the downward $\mathbf{E} \times \mathbf{B}$ drift in the northern hemisphere and, in contrast, the upward field-aligned plasma flow resisted the descent of the $F$ layer due to the downward $\mathbf{E} \times \mathbf{B}$ drift in the southern hemisphere.

From the examination of the meridional variation of ls-TEC and hs-TECs, the hemispherical asymmetry of ls-TEC and hs-TEC was ascribed to the field-aligned plasma flow combined with the vertical $\mathbf{E} \times \mathbf{B}$ drift and chemical recombination. The plasma flow was due to the ion drag of the thermospheric neutral wind, which was directed northward in most of the hours until $\sim 24 \mathrm{LT}$.

\section{Summary}

The thin-shell approximation of the ionosphere is a widely used technique to estimate ionospheric TEC from the trans-ionospheric radio waves from GNSS satellites. In most cases, single-shell approaches are taken, assuming a fixed shell height. Ionosphere pierce points of the slant ray path vary depending on the shell height. In spite of this, there is no clear criterion for choosing this height, and furthermore, the ionospheric height changes widely with the time and place. As a result, the vertical TEC and instrumental biases, which are simultaneously determined, depend on the shell height. To overcome this difficulty, a new technique was developed by taking a double-thin-shell approach.

The vertically distributed electron density was parameterized by two thin-shell layers. The spatiotemporal variation of TEC (strictly speaking, partial electron content) associated with each shell was approximated by the functional fitting of spherical surface harmonics. For this application, data set were prepared for 24 hours by the receiver network along the meridian at the southeast Asian longitude across the magnetic equator.

To evaluate the performance of the double-shell model, a single-shell model, which was a reduced version of the double-shell model, was also examined. The technique was first validated for a simulated slant TEC using the IRI electron density profile and artificially added random biases. The vertical TEC estimated from the slant TEC was compared with the known values of vertical TEC directly calculated by IRI (IRI-direct TEC). The single-shell model yielded different TECs and biases depending on the shell height. When the shell height was small, TEC was generally low and vice versa. Regardless of the shell height, the equatorial anomaly was flattened compared with the IRI-direct TEC. On the other hand, the double-shell approach successfully reproduced the IRI-direct TEC and randomly added arc biases. Also, the estimated vertical TEC and arc biases were independent of the shell height. 
In the application to real data obtained by the receiver network, the general characteristics of the difference between the single- and double-shell models were similar to those in the application to the IRI simulations. The double-shell results provided not only accurate TEC values but also comprehensive information on the ionospheric dynamics, such as the vertical ionospheric drift induced by the zonal electric field and the field-aligned plasma flow induced by the thermospheric neutral drag, that was not extracted by single-shell approaches.

\section{List of abbreviations}

GIM: Global ionosphere map

GNSS: Global Navigation Satellite Systems

GPS: Global Positioning System

IGRF: International Geomagnetic Reference Field

IPP: Ionospheric piece point

IRI: International Reference lonosphere

RINEX: Receiver Independent Exchange Format

RMSE: Root mean square error

TEC: Total electron content

Is-TEC: Lower-shell TEC

hs-TEC: Higher-shell TEC

\section{Availability of data and materials}

The GPS data used for this study were obtained from the IGS data center, the SEALION database, NICT/KMITL, and Department of Public Works and Town \& Country Planning (DPT), Thailand. The datasets used and analyzed during the current study are available from the corresponding author on reasonable request.

Competing interests

The authors declare that they have no competing interests.

Funding

The work in NAO, Chinese Academy of Sciences was supported by the National Natural Science Foundation of China (Grant Nos. 12073049, U2031146 and 11503040) and the National Key Research Program of China

"Collaborative Precision Positioning Project" (No. 2016YFB0501900). KH was partially supported by JSPS

KAKENHI Grant Number 20H00197. The work in KMITL was supported by King Mongkut's Institute of

Technology Ladkrabang Research Fund KREF206311.

Authors' contributions

TM developed the model. KH, GM, PS, and QW gathered and processed the RINEX data.

Acknowledgements

The work was partially carried out when TM was staying in KMITL, Thailand under the KMITL melting pot fund.

The authors are grateful to the members of the SEALION project for obtaining data.

\section{Author details}

${ }^{1}$ National Astronomical Observatories, Chinese Academy of Sciences, 20A, Datun Road., Chaoyang District, 100012 Beijing, China. ${ }^{2}$ National Institute of Information and Communications Technology, 2-1 Nukuikita 4-chome, 184-8795 Tokyo, Japan. ${ }^{3}$ School of Engineering, King Mongkut's Institute of Technology Ladkrabang, Chalongkrung Rd, Ladkrabang, 10520 Bangkok, Thailand.

\section{References}

Arikan et al.(2008). Arikan F, Nayir H, Sezen U, Arikan O (2008) Estimation of single station interfrequency receiver bias using GPS-TEC. Radio Science 43: RS4004. doi:10.1029/2007RS003785

Bilitza et al.(2017). Bilitza D, Altadill D, Truhlik V, Shubin V, Galkin I, Reinish B, Huang X (2017) International Reference lonosphere 2016: From ionospheric climate to real-time weather predictions. Space Weather 15: 418-429. doi:10.1002/2016SW001593

Brunini et al.(2005). Brunini C, Meza A, Bosch W (2005) Temporal and spatial variability of the bias between TOPEX- and GPS-derived total electron content. Journal of Geodesy 79: 175-188. doi:10.1007/s00190-005-0448-z

Choi et al.(2011). Choi BK, Cho JH, Lee SJ (2011) Estimation and analysis of GPS receiver differential code biases using KGN in Korean Peninsula. Advances in Space Research 47: 1590-1599. doi:10.1016/j.asr.2010.12.021

Choi et al.(2013). Choi BK, Park JU, Roh KM, Lee SJ (2013) Comparison of GPS receiver DCB estimation methods using a GPS network. Earth Planets and Space 65: 707-711. doi:10.5047/eps.2012.10.003

Fejer et al.(2008). Fejer BG, Jensen JW, Su S-Y (2008) Quiet time equatorial $F$ region vertical plasma drift model derived from ROCSAT-1 observations. Journal of Geophysical Research 113: A05304. doi:10.1029/2007JA012801 Haykin(1994). Haykin S (1994) Neural networks - A comprehensive foundation, Macmillan College Publishing Company, Inc.

Hernández-Pajares et al.(1999). Hernández-Pajares M, Juan JM, Sanz J (1999) New approaches in global ionospheric determination using ground GPS data. Journal of Atmospheric and Solar-Terrestrial Physics 61: 1237-1247 
Hernández-Pajares et al.(2009). Hernández-Pajares M, Juan JM, Sanz J, Orus R, Garcia-Rigo A, Feltens J, Komjathy A, Schaer SC, Krankowski A (2009) The IGS VTEC maps: a reliable source of ionospheric information since 1998. Journal of Geodesy 83: 263-275. doi:10.1007/s00190-008-0266-1

Klobuchar(1987). Klobuchar J (1987) lonospheric time-delay algorithms for single-frequency GPS users. IEEE Transactions on Aerospace and Electronic Systems 3: 325-331

Lanyi and Roth(1988). Lanyi GE, Roth T (1988) A comparison of mapped and measured total ionospheric electron content using global positioning system and beacon satellite observations. Radio Science 23(4): 483-492

Ma and Maruyama(2003). Ma G, Maruyama T (2003) Derivation of TEC and estimation of instrumental biases from GEONET in Japan. Annales Geophysicae 21: 2083-2093

Ma et al.(2005). Ma XF, Maruyama T, Ma G (2005) Determination of GPS receiver differential biases by neural network parameter estimation method. Radio Science 40: RS1002. doi:10.1029/2004RS003072

Mannucci et al.(1998). Mannucci AJ, Wilson BD, Yuan DN, Ho CH, Lindqwister UJ, Runge TF (1998) A global mapping technique for GPS-derived ionospheric total electron content measurements. Radio Science 33(3): $565-582$

Otsuka et al.(2002). Otsuka Y, Ogawa T, Saito A, Tsugawa T, Fukao S, Miyazaki S (2002) A new technique for mapping of total electron content using GPS network in Japan. Earth Planets and Space 54: 63-70

Prasad et al.(2016). Prasad R, Kumar S, Jayachandran PT (2016) Receiver DCB estimation and GPS vTEC study at a low latitude station in the South Pacific. Journal of Atmospheric and Solar-Terrestrial Physics 149: 120-130

Thébault et al.(2015). Thébault E et al. (2015) International Geomagnetic Reference Field: the 12th generation. Earth Planets and Space 67: 79

Yang and Tseng(1996). Yang S-S, Tseng C-S (1996) An orthogonal neural network for function approximation. IEEE Transactions on Systems, Man, and Cybernetics-Part B: Cybernetics 26(5): 779-785

Zhang et al.(2009). Zhang W, Zhang DH, Xiao Z (2009) The influence of geomagnetic storms on the estimation of GPS instrumental biases. Annales Geophysicae 27: 1613-1623

\section{Figures}

Figure 1 Geometry of double-shell approximation of ionosphere. The azimuth $(\gamma)$ and elevation $(\eta)$ angles of the satellite ray path and the differential delay $(\Delta \tau)$ between two signals are measured. The ray path crosses shells at the height $h_{s i}(i=L o$ and $H i$ for the lower and higher shells, respectively). The point of intersection is referred to as the ionosphere pierce point (IPP). $\mathrm{RX}$ is the receiver.

Figure 2 Orthogonal network for functional fitting. Each node of the orthogonal layer calculates a component of the surface harmonics. The weighted sum of the outputs of the orthogonal layer for each shell is further transferred by the function $\hat{\sigma}$ in the output layer. The sum of the two shells gives the vertical TEC. Weights and biases are adjusted to minimize the residual error.

Figure 3 Activation function in output layer. 
Figure 4 IPPs observed by eight GNSS receivers (open circles) in the period from 22 UT, 16 March 2014 to 22 UT, 17 March 2014. Orange dots indicate IPPs at a shell height of $300 \mathrm{~km}$ and green dots indicate at a shell height of $600 \mathrm{~km}$.

Figure 5 TEC maps. (a) Vertical TEC directly calculated from IRI model (IRI-direct TEC), (b) (d) vertical TEC reconstructed by single-shell model for shell heights of 350,450 , and $550 \mathrm{~km}$, and $(\mathrm{e})-(\mathrm{g})$ error of single-shell model reconstruction for each shell height. The dash-dotted line indicates the magnetic equator.

Figure 6 Estimation errors of arc biases. (a)-(c) Single-shell model with shell heights of 350, 450, and $550 \mathrm{~km}$. (d)-(f) Double-shell model with combinations of shell heights of 250/600,300/600, and $300 / 700 \mathrm{~km}$ for lower/higher shells.

Figure 7 Functional fitting error, RMSE of bias estimation, and RMSE of slant TEC for different shell heights.

Figure 8 TEC maps. (a)-(c) Vertical TEC reconstructed by double-shell model for shell height combinations of $250 / 600,300 / 600$, and $300 / 700 \mathrm{~km}$ for lower/higher shells, and (d)-(f) TEC error for double-shell model reconstruction for each shell height combination.

Figure 9 TEC maps derived from actual observations by double-shell model. (a)-(c) Vertical TEC, Is-TEC, and hs-TEC for shell height combination of 300 and $600 \mathrm{~km}$. The red dashed line indicates a magnetic latitude of $12^{\circ} \mathrm{N}$. (d)-(f) Vertical TEC, Is-TEC, and hs-TEC for shell height combination of 280 and $700 \mathrm{~km}$.

Figure 10 TEC maps derived from actual observations by single-shell model. (a)-(c) Vertical TEC for shell heights of 350,450 , and $550 \mathrm{~km}$, and (d)-(f) difference between left panels and double-shell TEC with shell height combination of 300 and $600 \mathrm{~km}$.

Figure 11 Local time variations of Is-TEC, hs-TEC, and two-shell TEC, at magnetic equator (a) and away from equator at $12^{\circ} \mathrm{N}$ magnetic latitude (b) with shell height combination of 300 and $600 \mathrm{~km}$. The two latitudes are shown by the dash-dotted line (magnetic equator) and red dashed line $\left(12^{\circ} \mathrm{N}\right.$ magnetic latitude) in Figures $9 \mathrm{a}-9 \mathrm{c}$.

Figure 12 Latitudinal variation of Is-TEC, hs-TEC, and two-shell TEC with shell height combination of 300 and $600 \mathrm{~km}$. (a) Transition from 11 to $12 \mathrm{LT}$ and (b) transition from 17 to 18 LT, (c) 20 LT, and (d) 22 LT with plots of two-shell TEC for 20 LT (purple dotted line) and doubled hs-TEC (green dotted line) for convenience of comparison. 


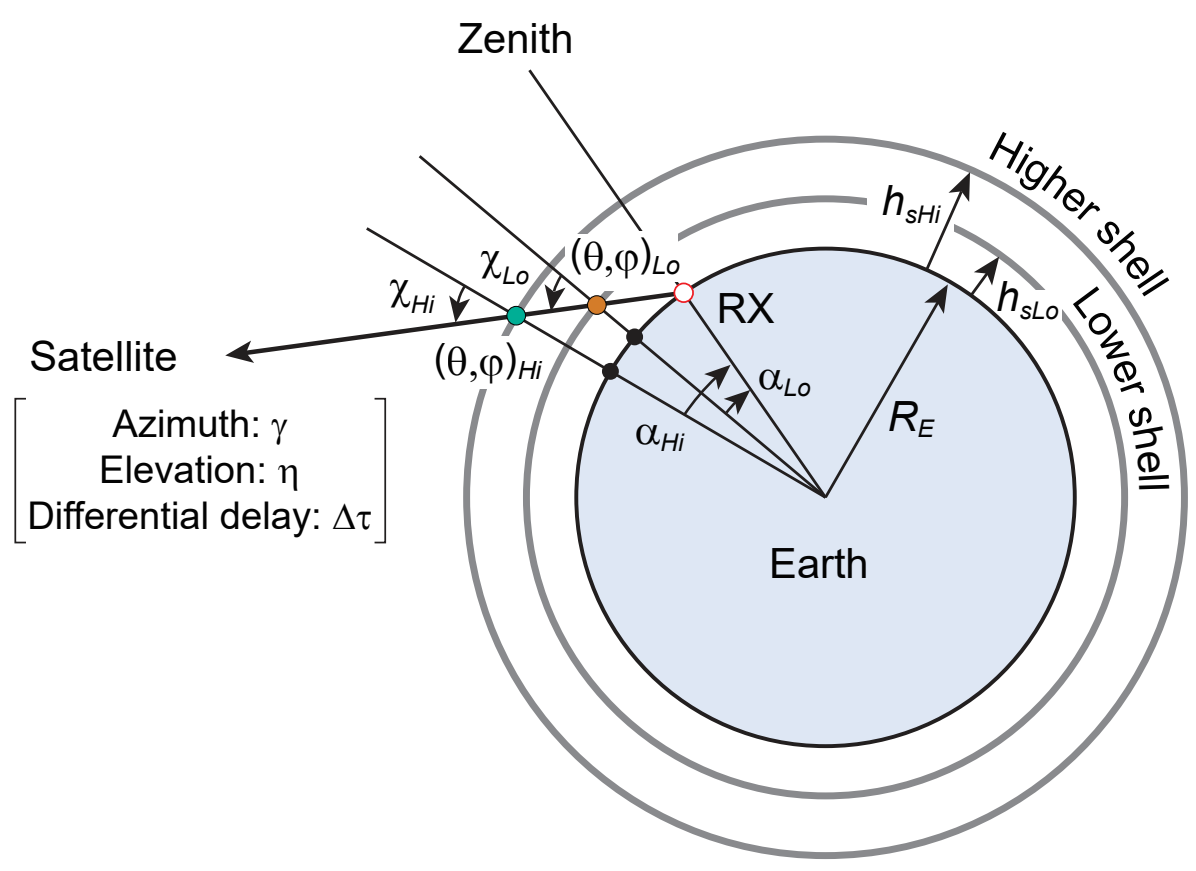

figure 1 


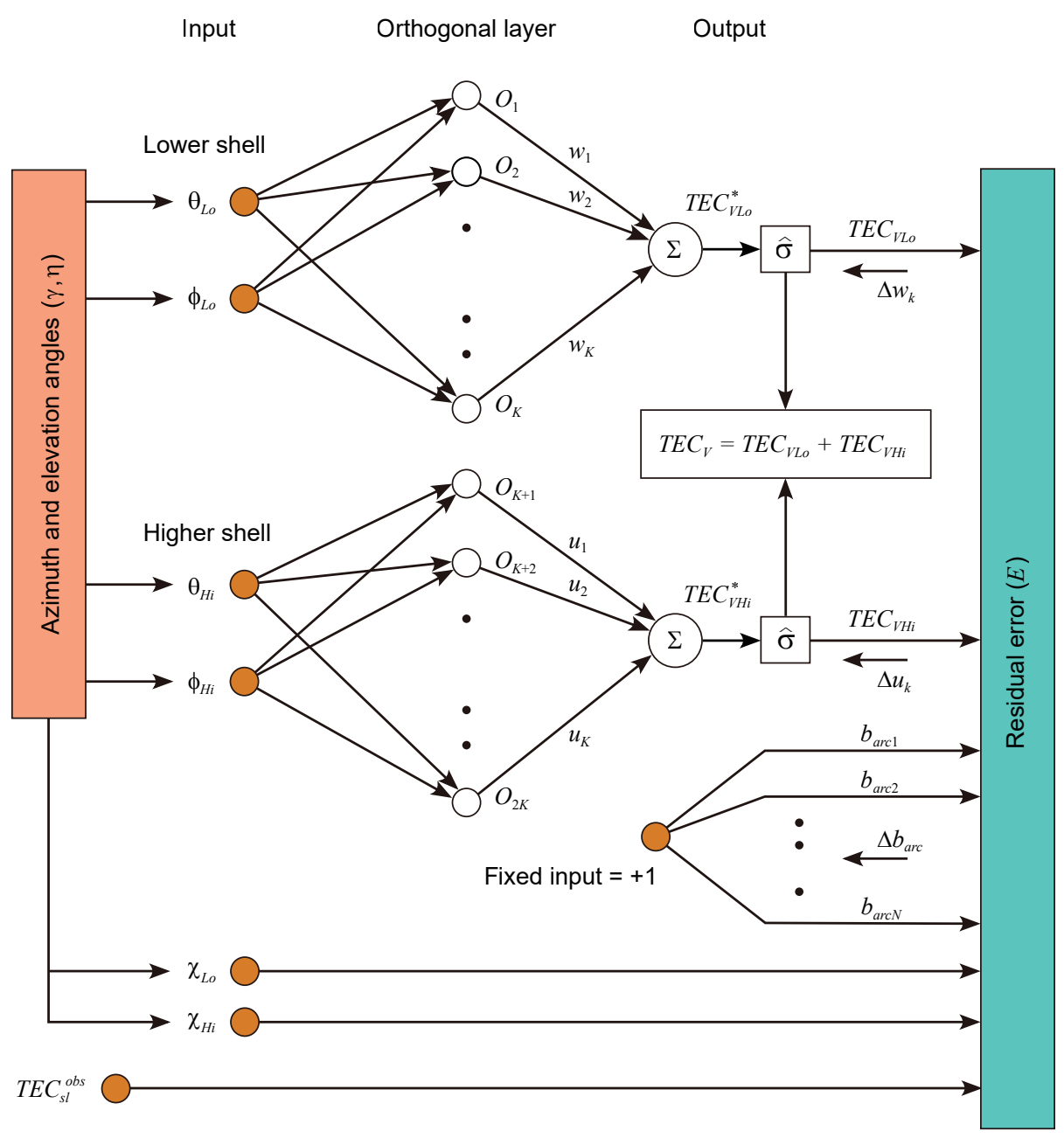

figure 2 


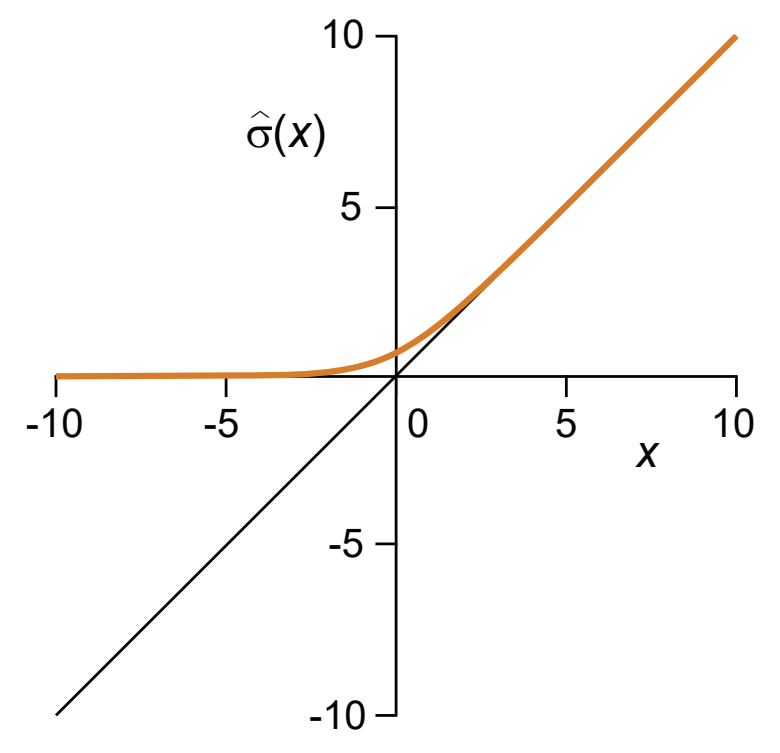

figure 3 


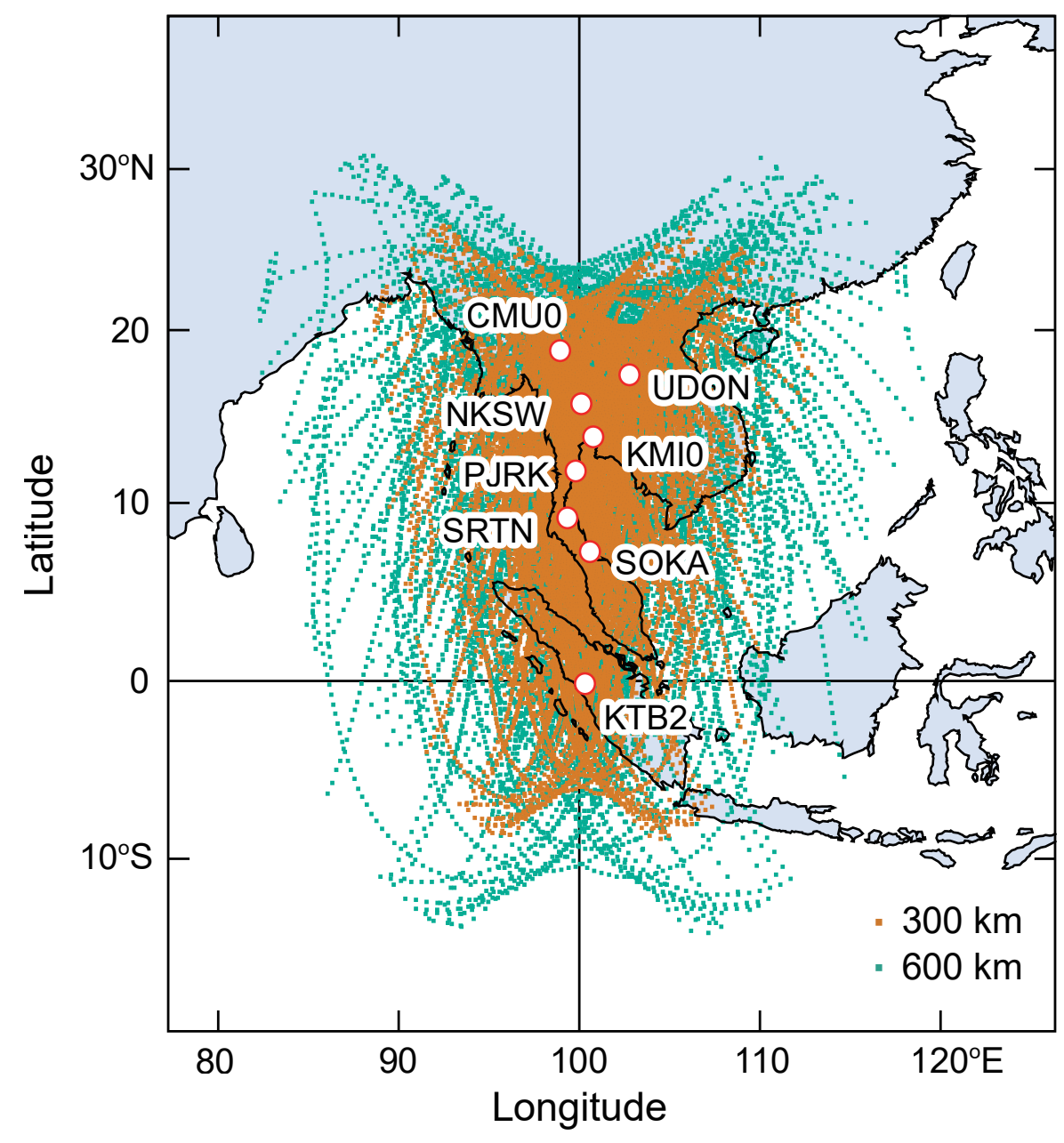

figure 4 


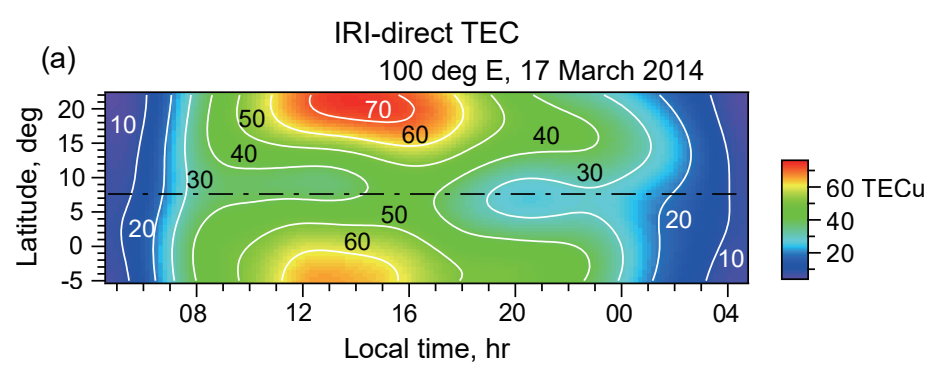

Single-shell model (IRI simulation)

(b)

TEC

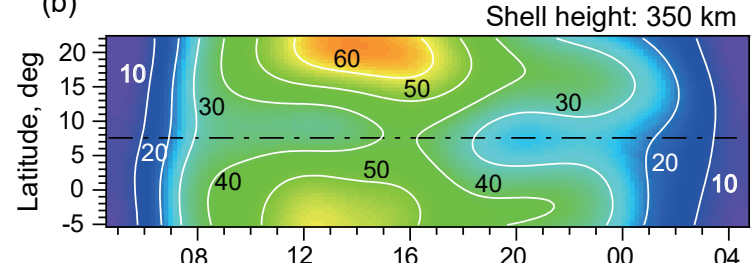

(c)

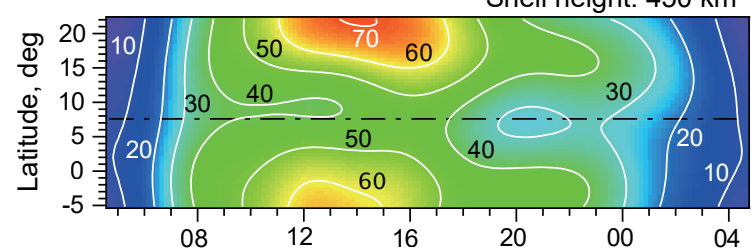

(d)

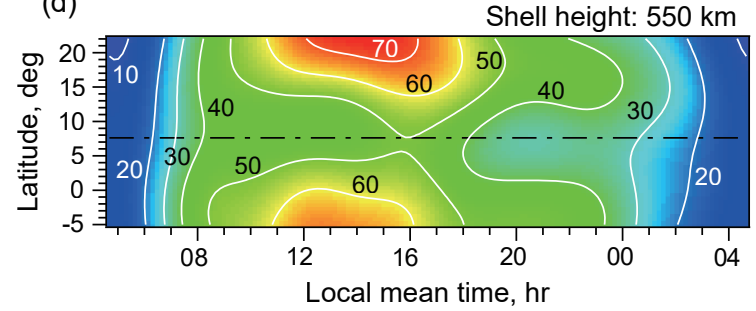

(e) $\begin{array}{ll} & 8 \mathrm{TTEC} \\ & 4 \\ = & 0 \\ - & -4 \\ -8 & \end{array}$

Error

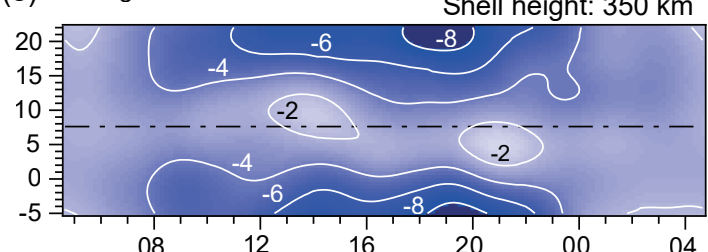

Shell height: $450 \mathrm{~km}$

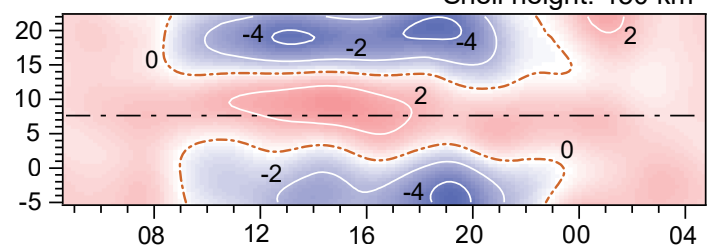

(g)

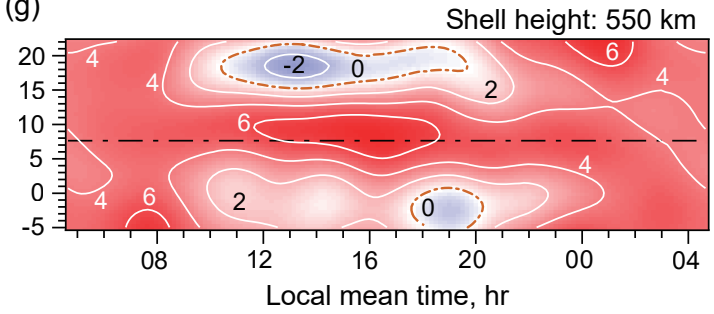

figure 5 
Single-shell model (IRI simulation)

(a)

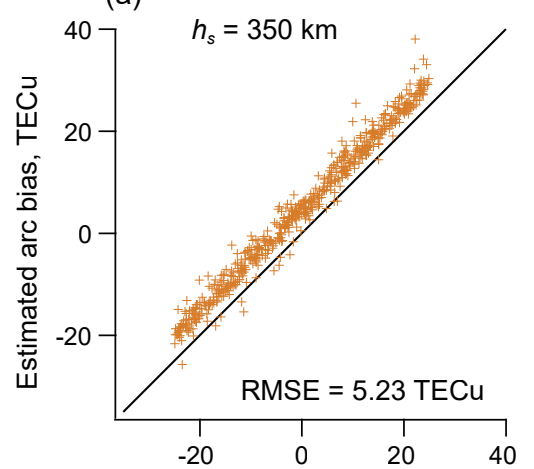

(b)

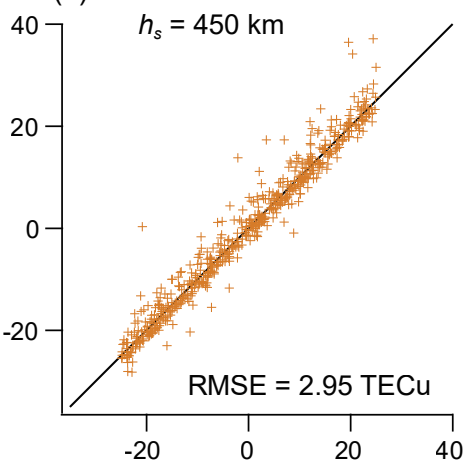

Randomly generated arc bias, TECu (c)

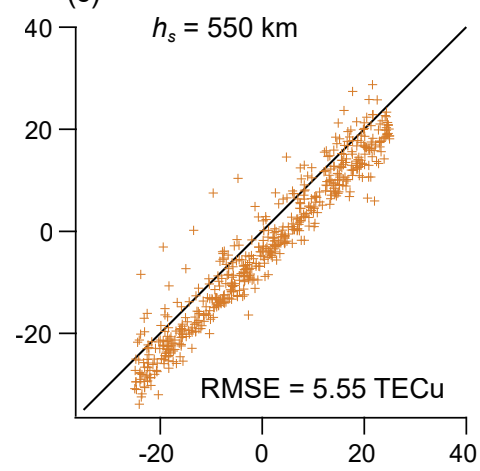

Double-shell model (IRI simulation)
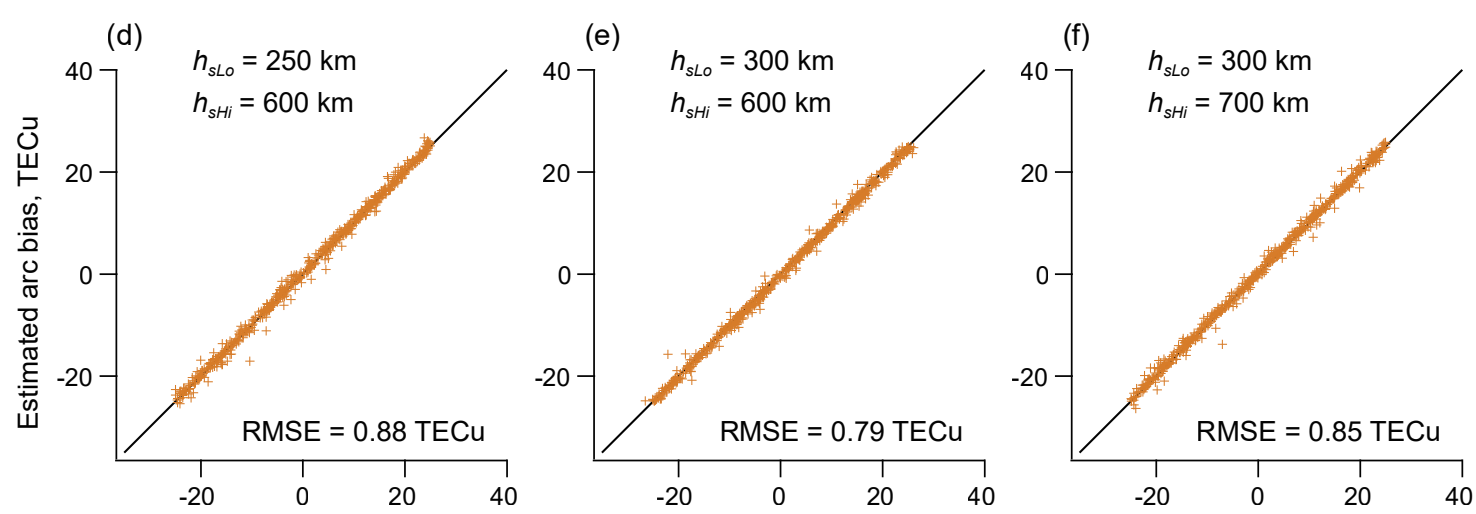

Randomly generated arc bias, TECu

\section{figure 6}




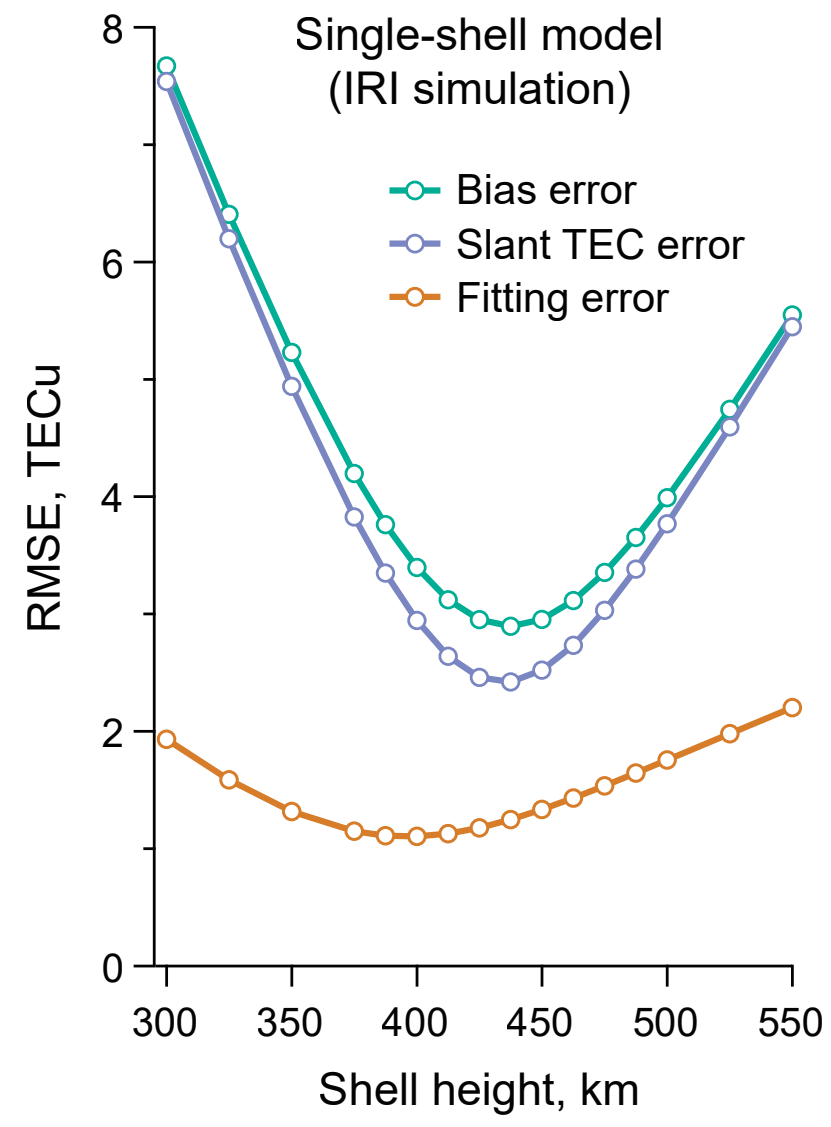

figure 7 
Double-shell model (IRI simulation)

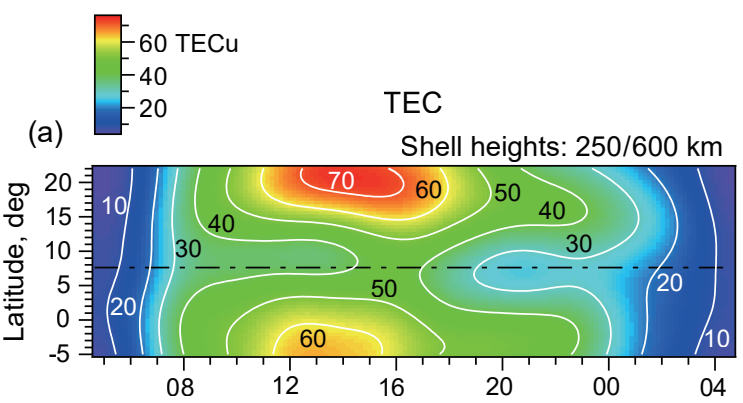

(b)

Shell heights: 300/600 km

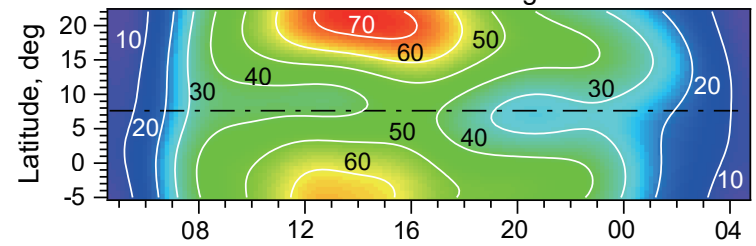

(c) Shell heights: $300 / 700 \mathrm{~km}$

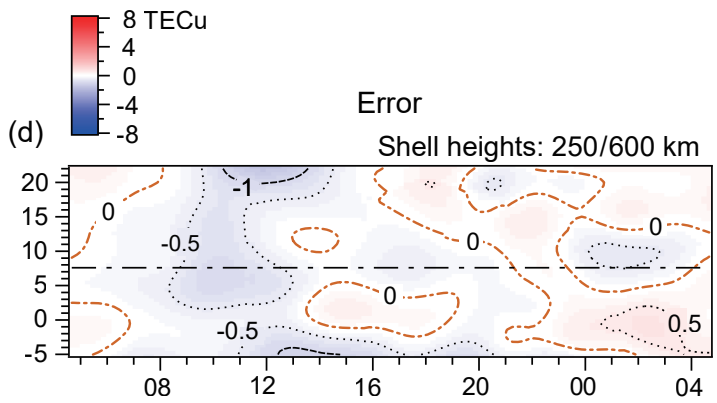

(e)

Shell heights: $300 / 600 \mathrm{~km}$

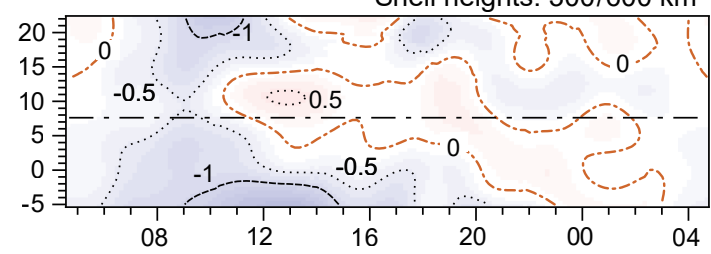

(f)

Shell heights: $300 / 700 \mathrm{~km}$
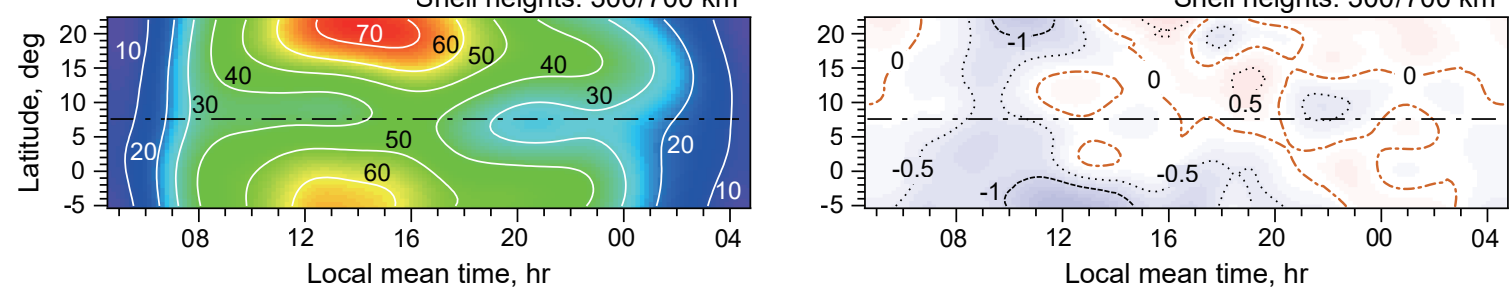

figure 8 

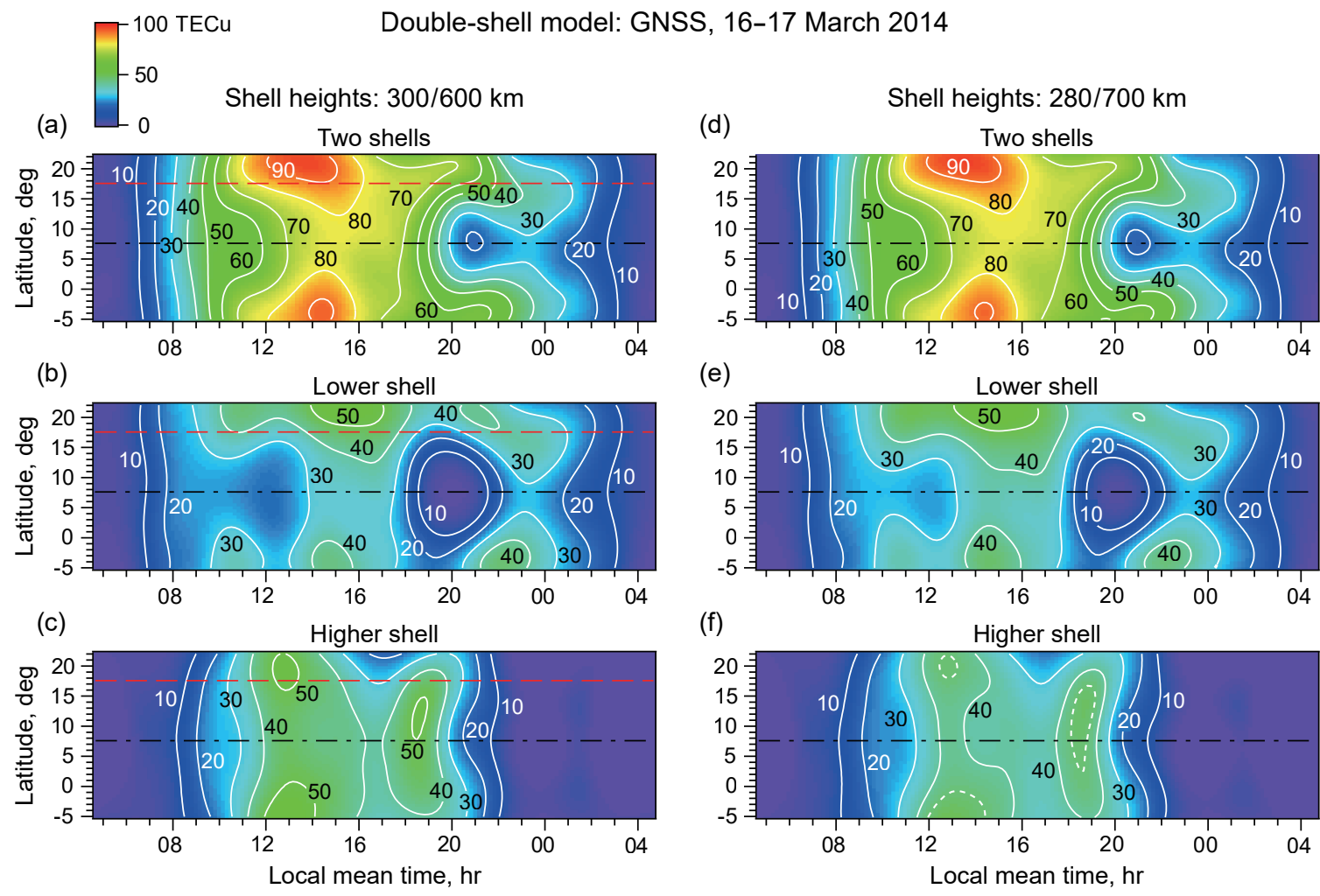

(e)

S, 16-17 March 2014
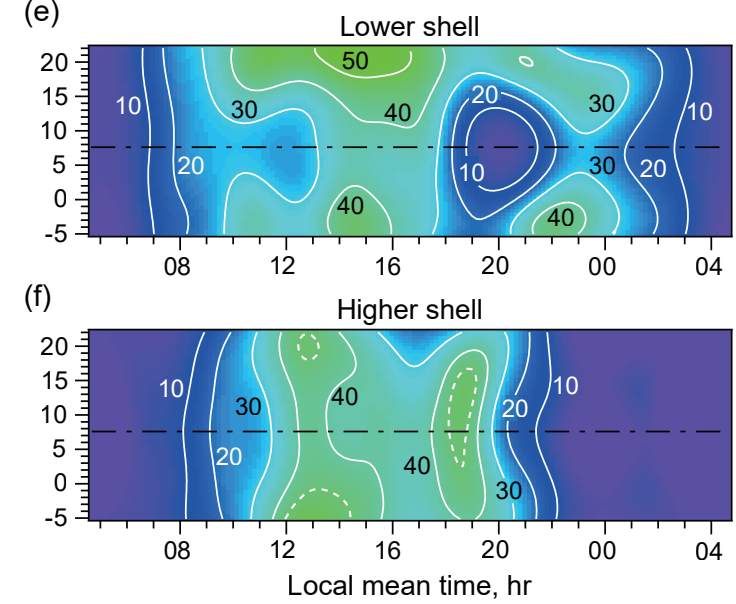

figure 9 
Single-shell model: GNSS, 16-17 March 2014
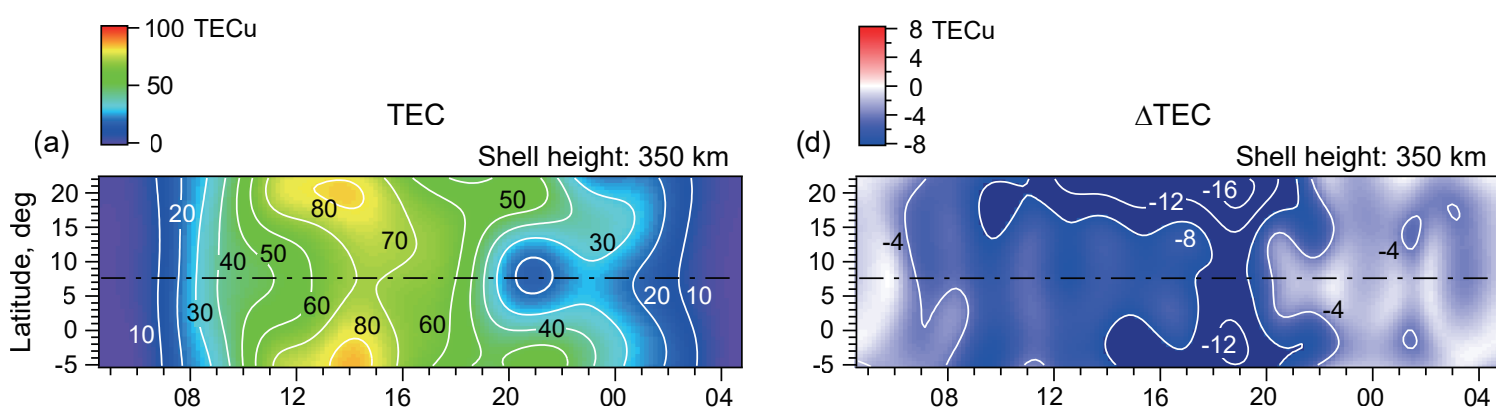

(b) Shell height: $450 \mathrm{~km}$

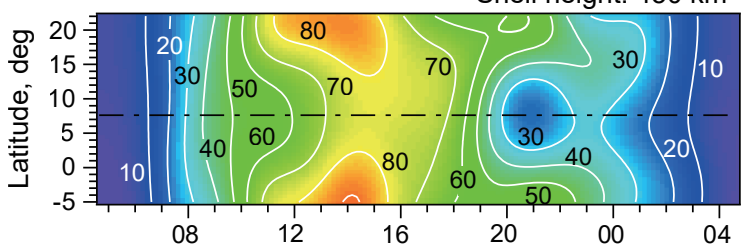

(c) Shell height: $550 \mathrm{~km}$
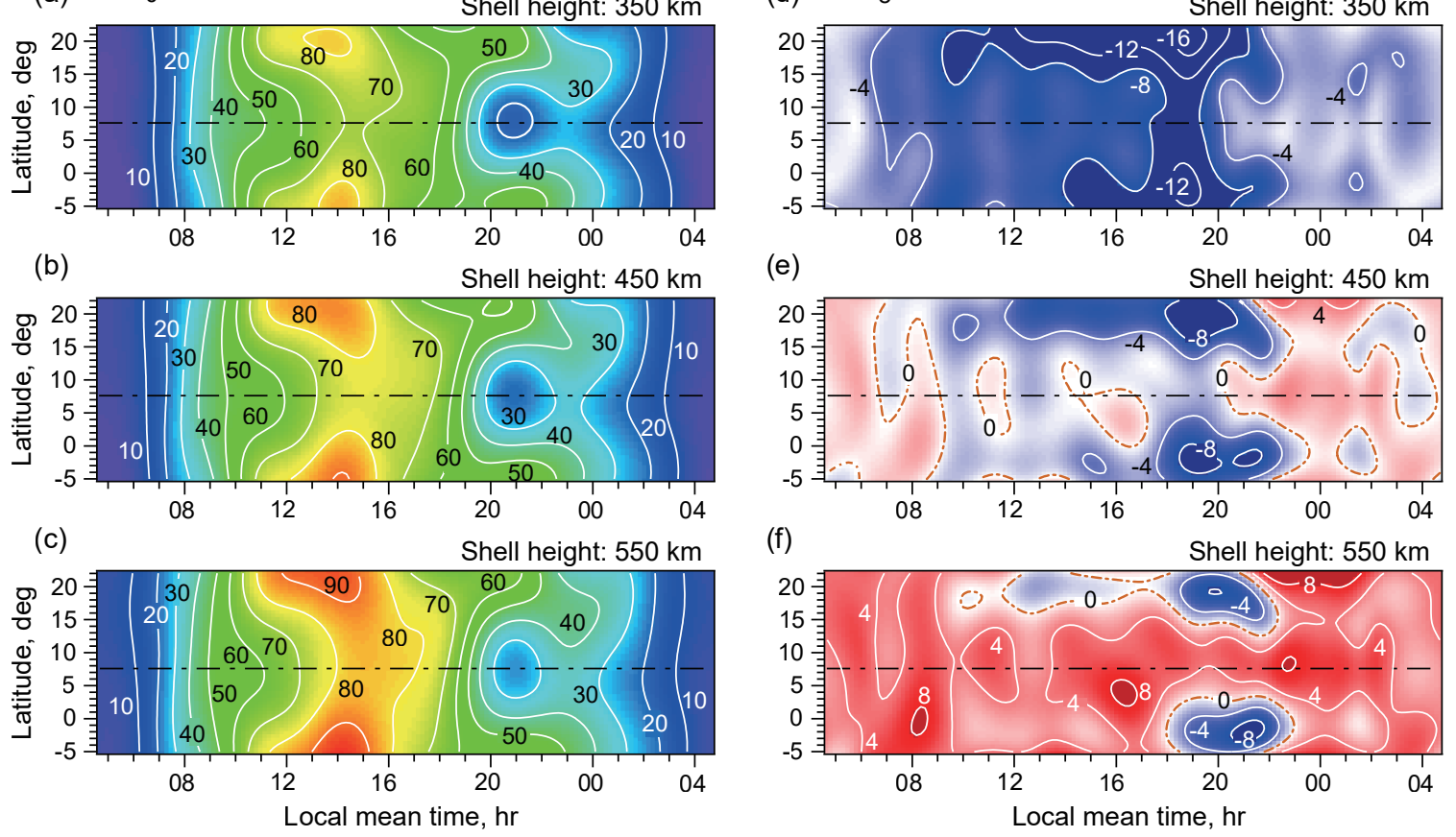

figure 10 
(a)
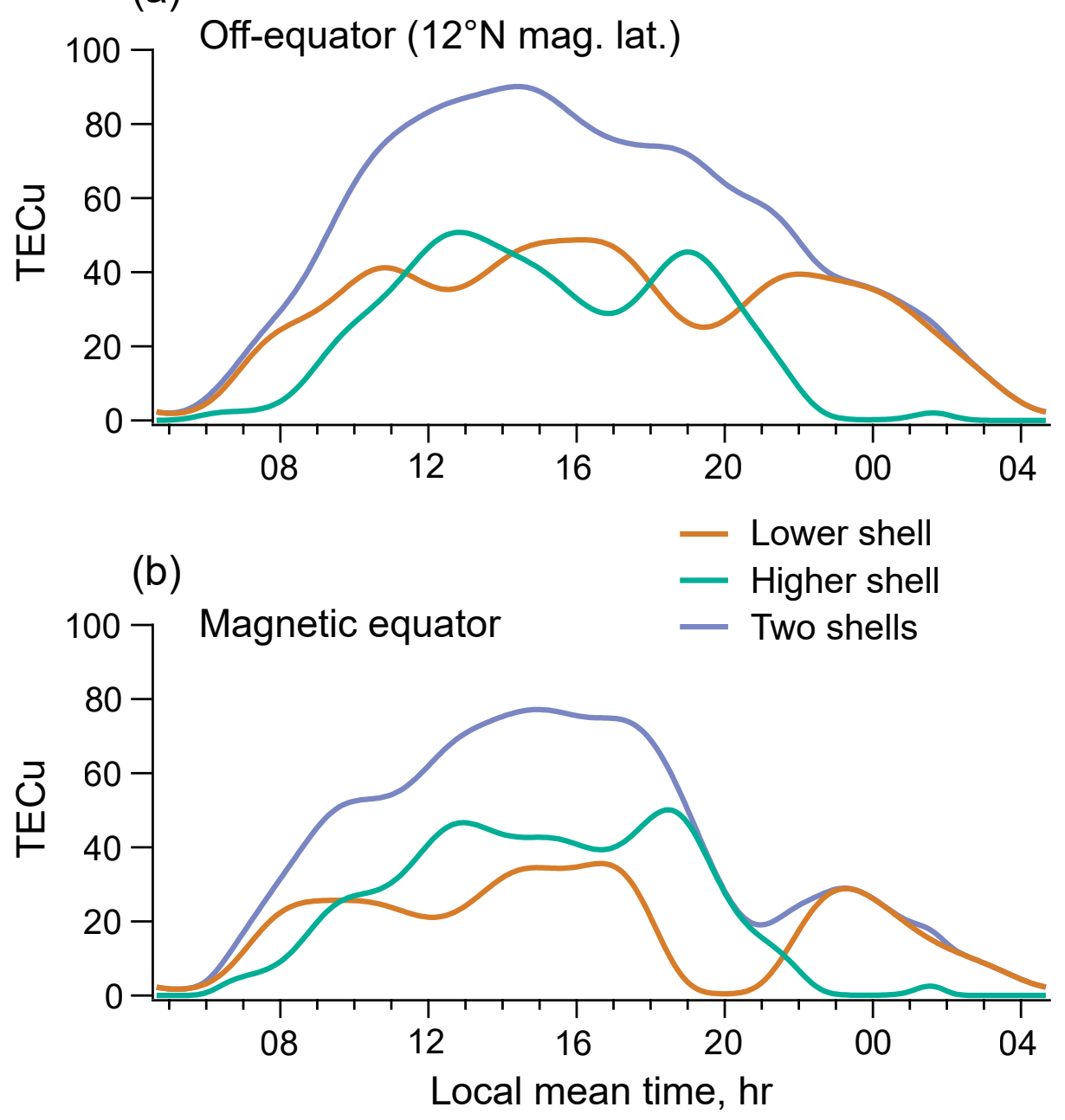

figure 11 
(a)

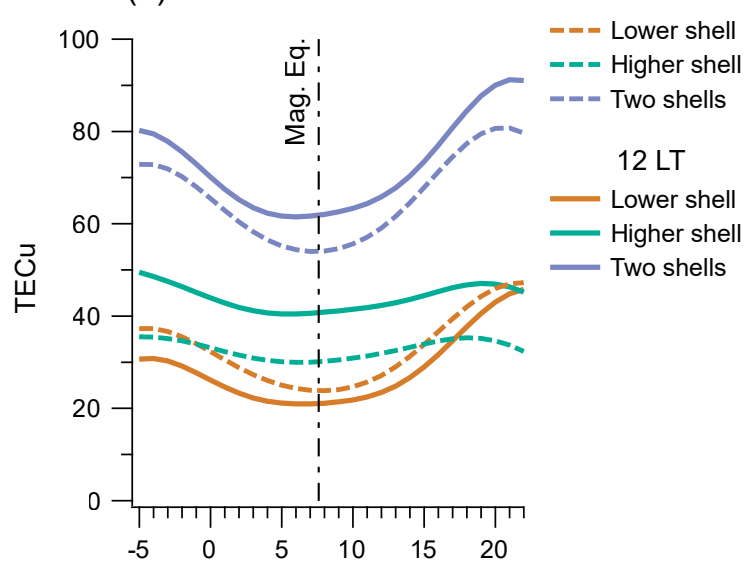

(c)

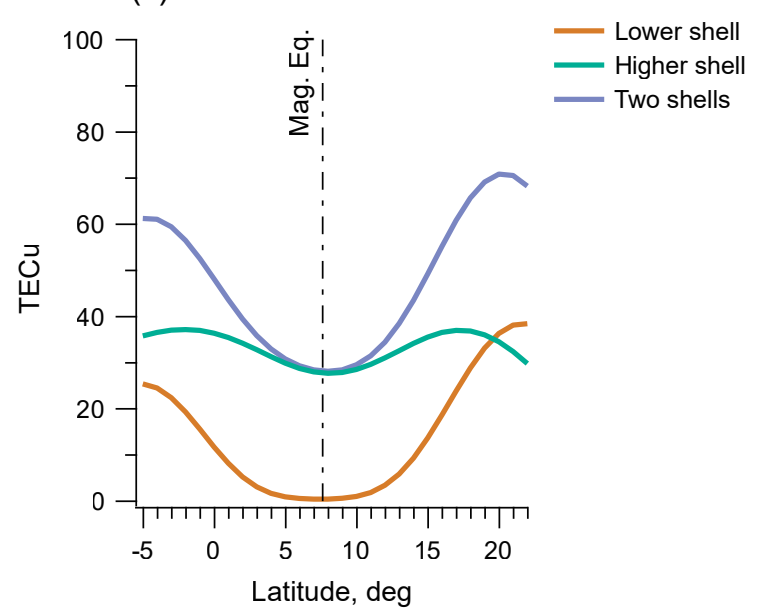

(b)

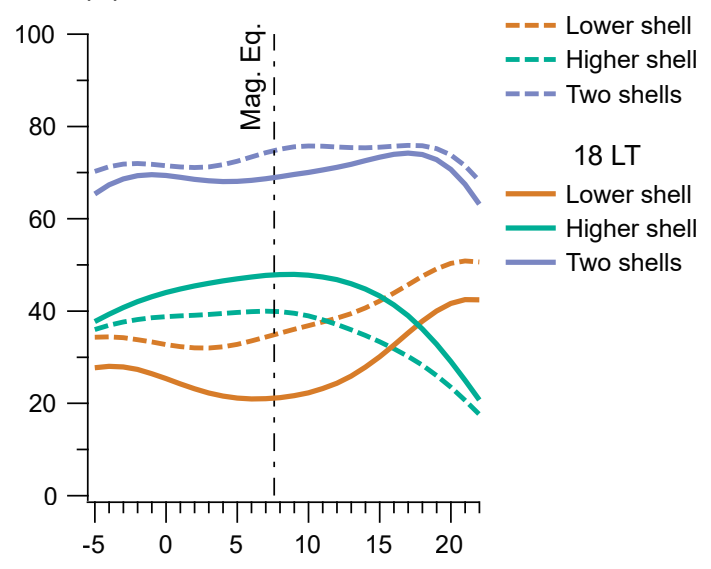

(d)

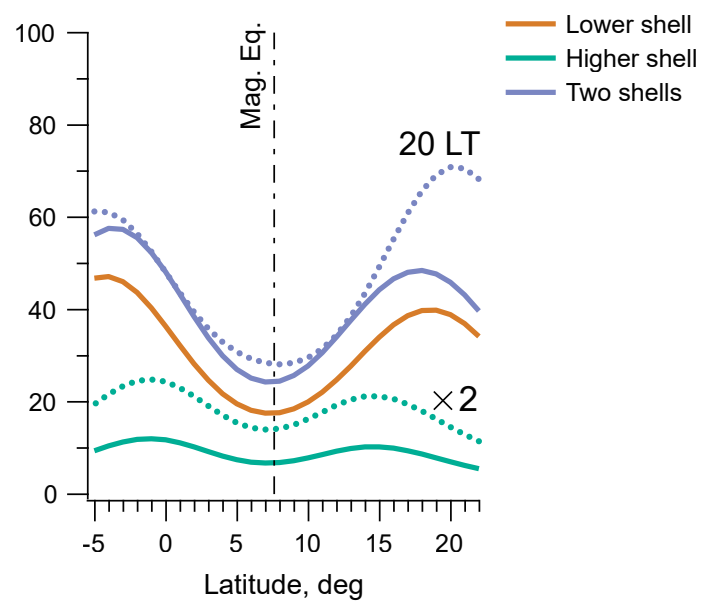

figure 12 


\section{Zenith}

Satellite

$\left[\begin{array}{c}\text { Azimuth: } \gamma \\ \text { Elevation: } \eta \\ \text { Differential delay: } \Delta \tau\end{array}\right]$

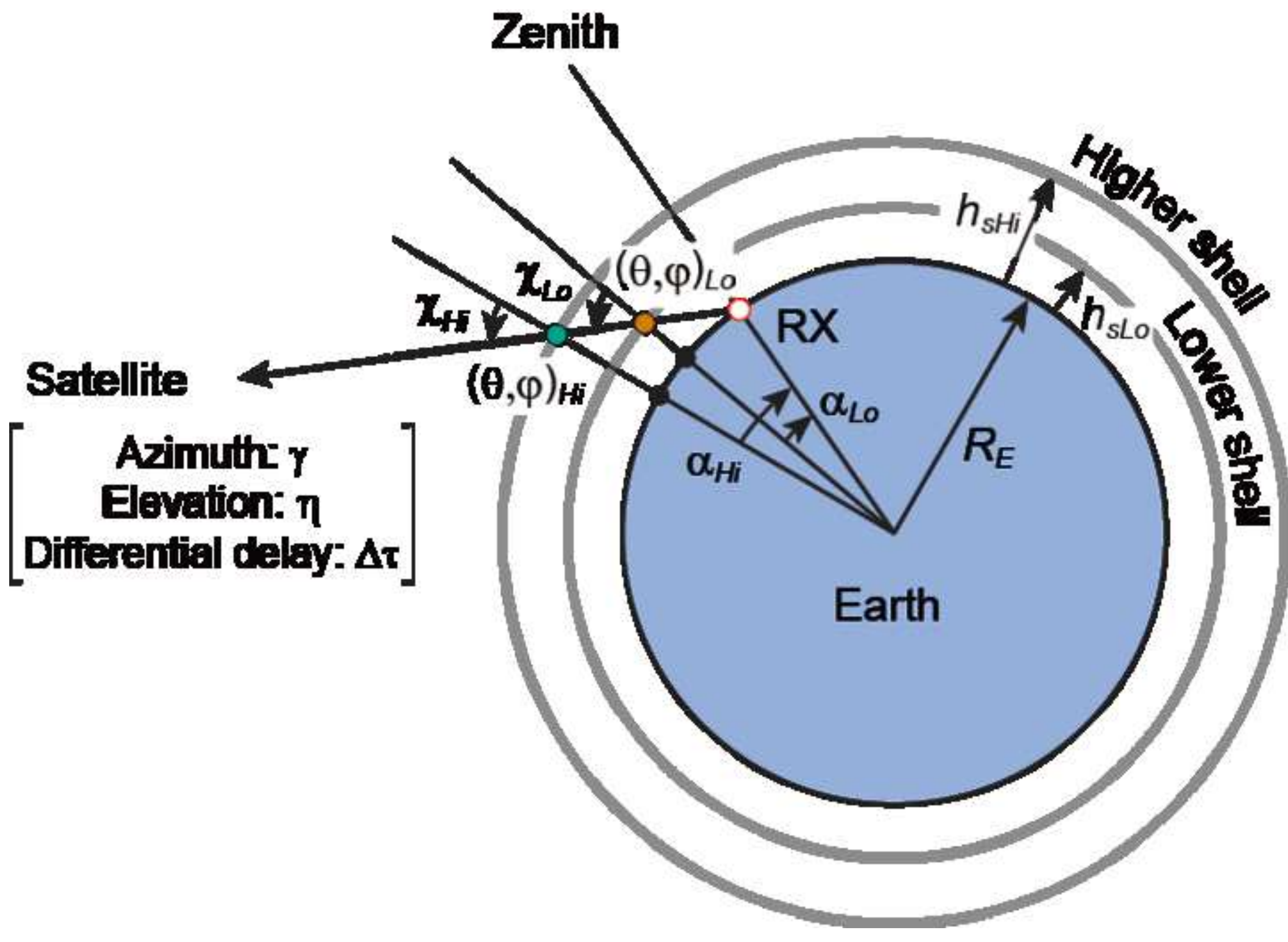


Figures

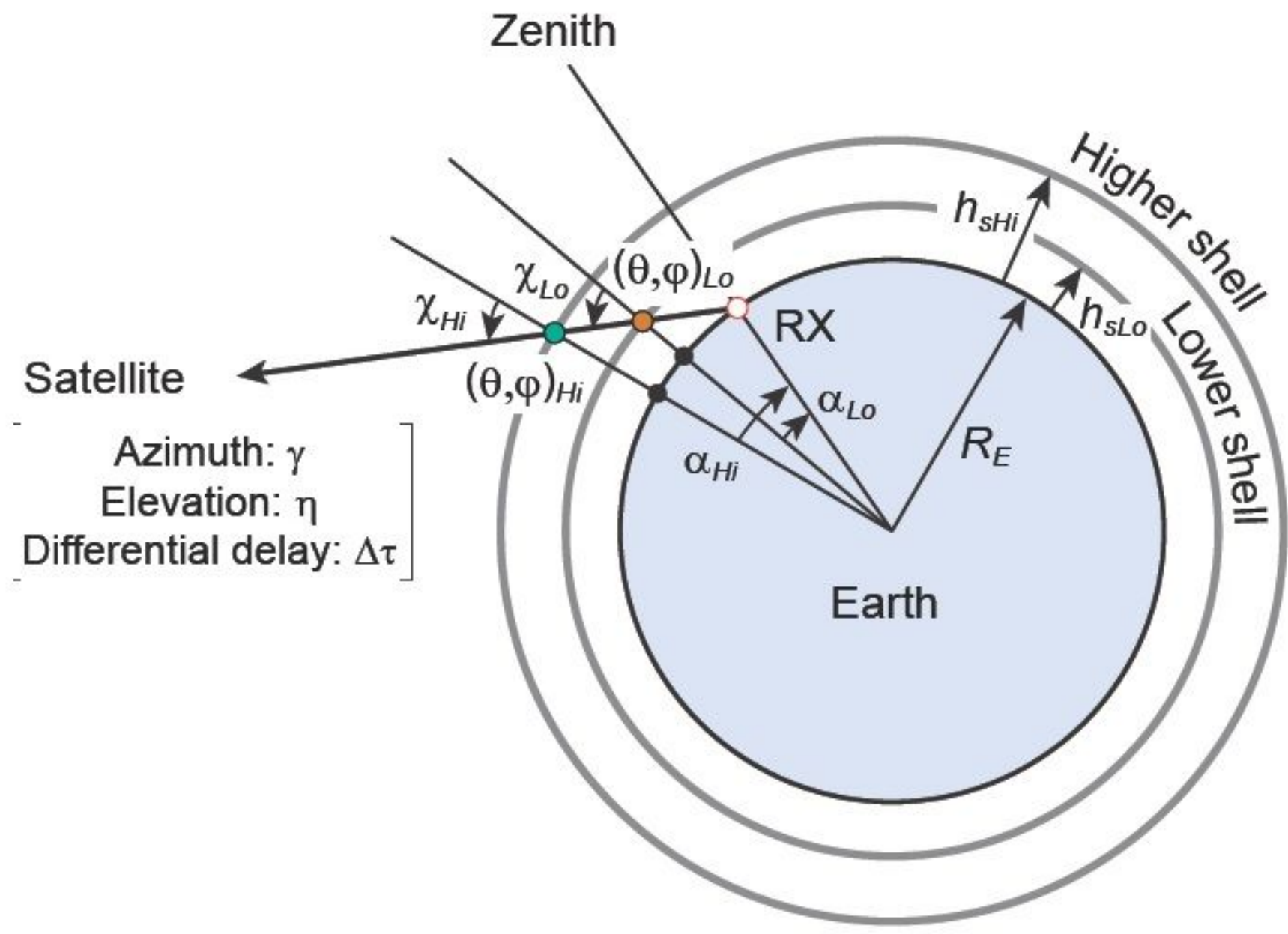

\section{Figure 1}

Geometry of double-shell approximation of ionosphere. The azimuth ( $\mathrm{\gamma}$ ) and elevation (n) angles of the satellite ray path and the differential delay $(\Delta T)$ between two signals are measured. The ray path crosses shells at the height hsi ( $\mathrm{i}=\mathrm{Lo}$ and $\mathrm{Hi}$ for the lower and higher shells, respectively). The point of intersection is referred to as the ionosphere pierce point (IPP). RX is the receiver. 


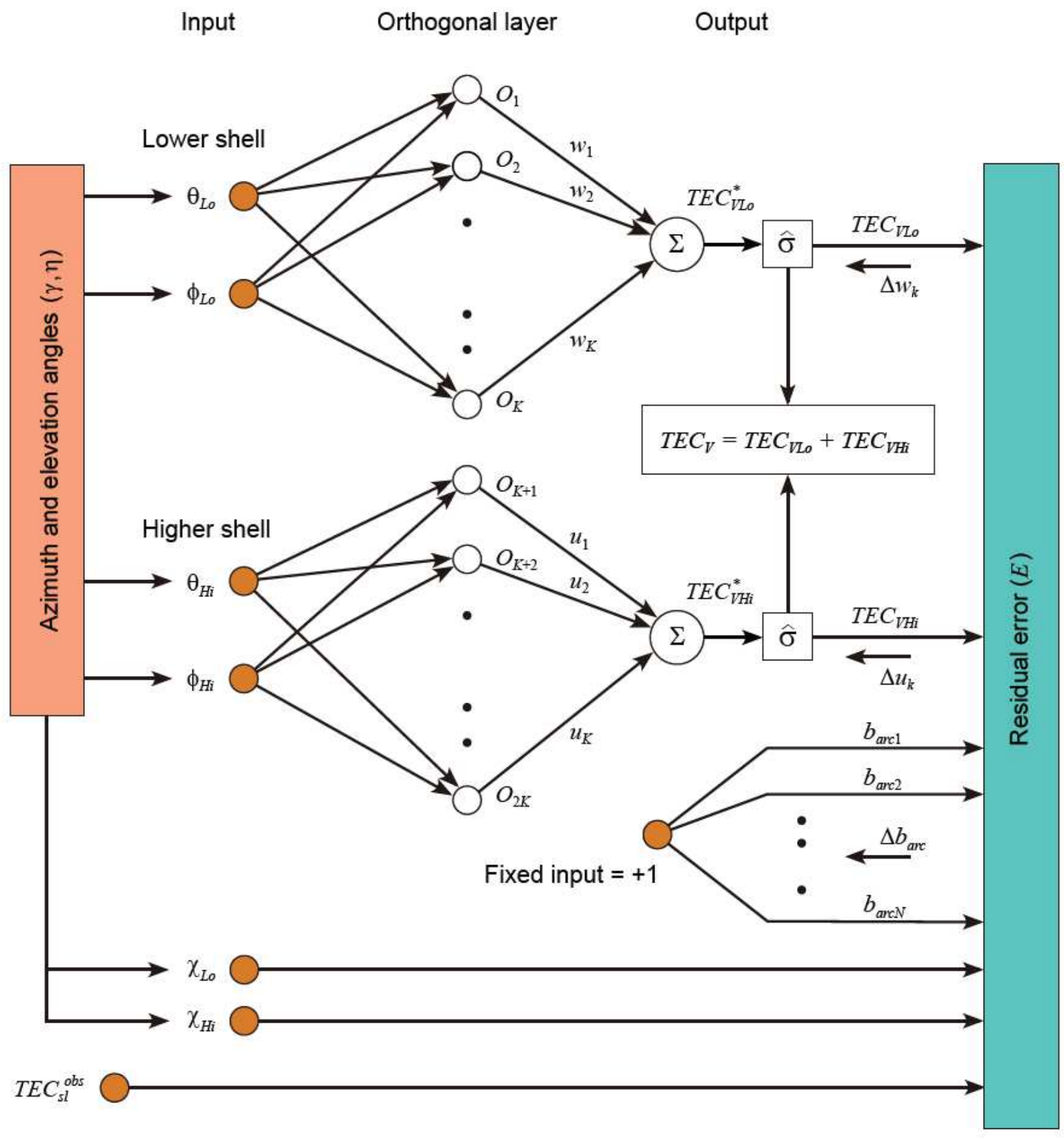

\section{Figure 2}

Orthogonal network for functional fitting. Each node of the orthogonal layer calculates a component of the surface harmonics. The weighted sum of the outputs of the orthogonal layer for each shell is further transferred by the function $\sigma^{\wedge}$ in the output layer. The sum of the two shells gives the vertical TEC. Weights and biases are adjusted to minimize the residual error. 


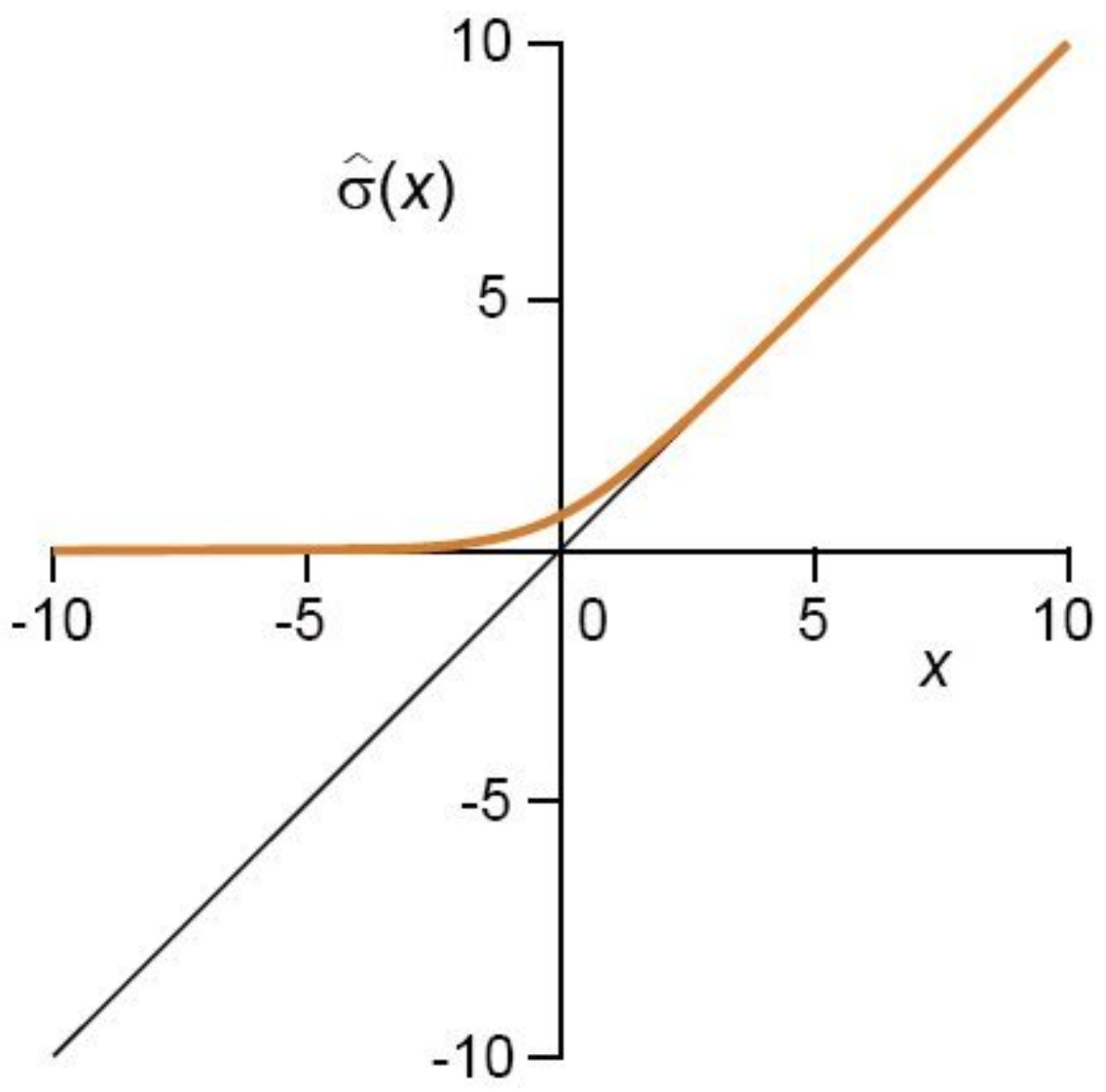

Figure 3

Activation function in output layer. 


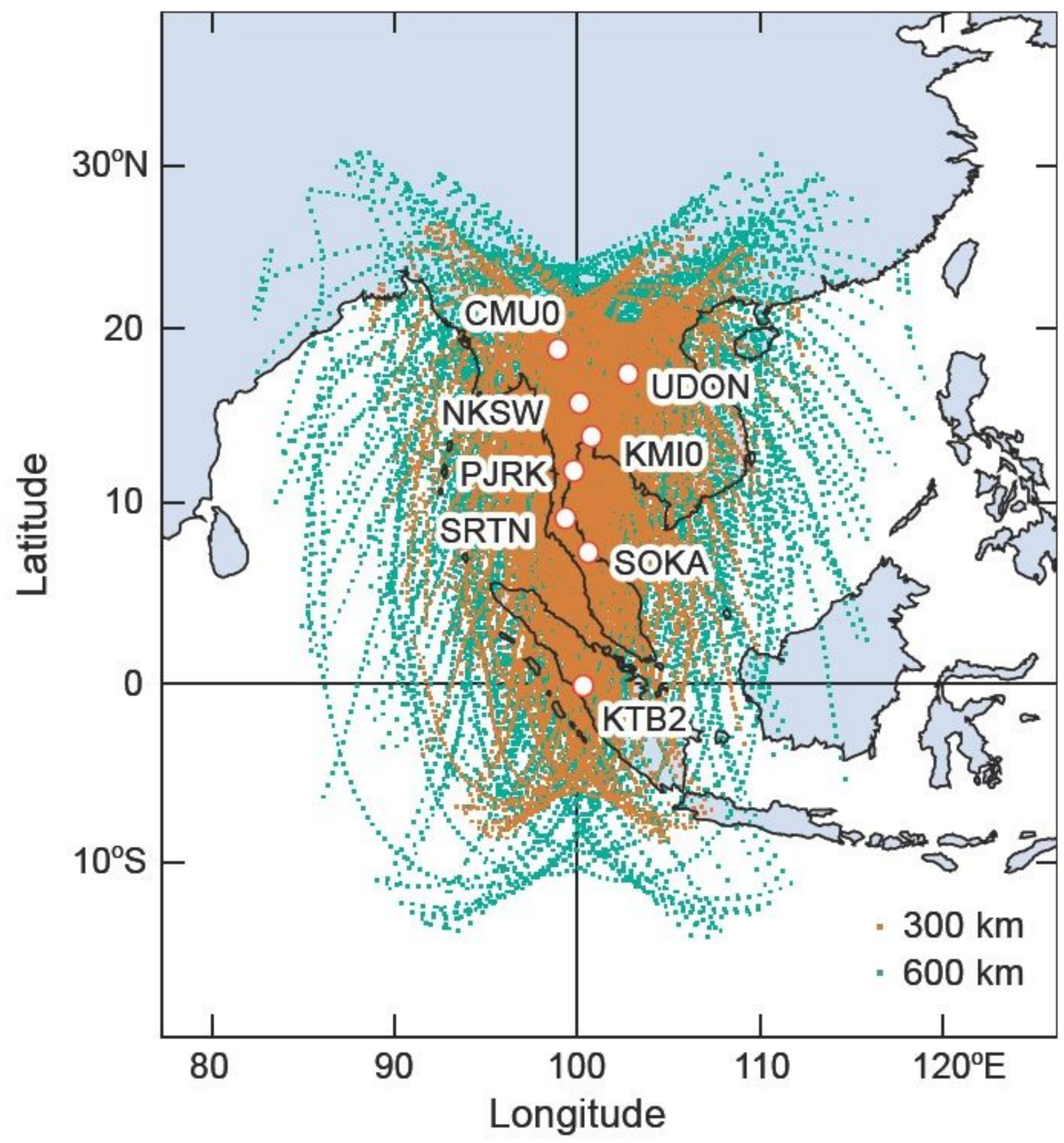

Figure 4

IPPs observed by eight GNSS receivers (open circles) in the period from 22 UT, 16 March 2014 to 22 UT, 17 March 2014. Orange dots indicate IPPs at a shell height of $300 \mathrm{~km}$ and green dots indicate at a shell height of $600 \mathrm{~km}$. 
(a) IRI-direct TEC

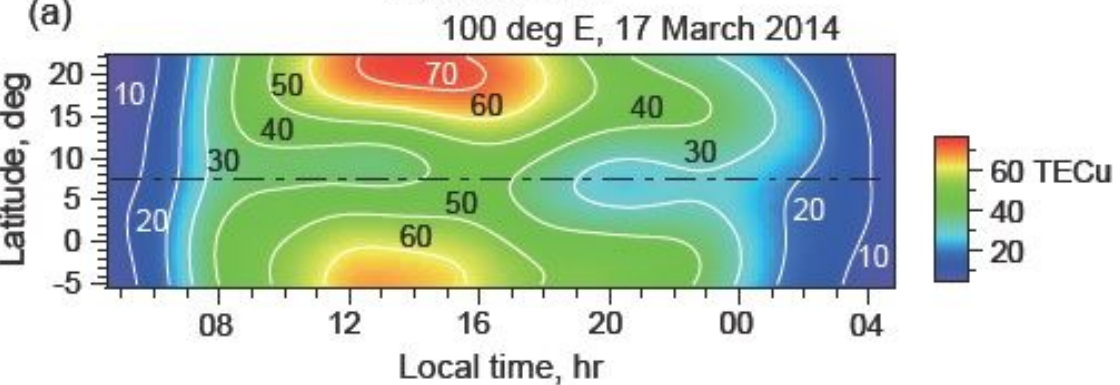

Single-shell model (IRI simulation)

(b)

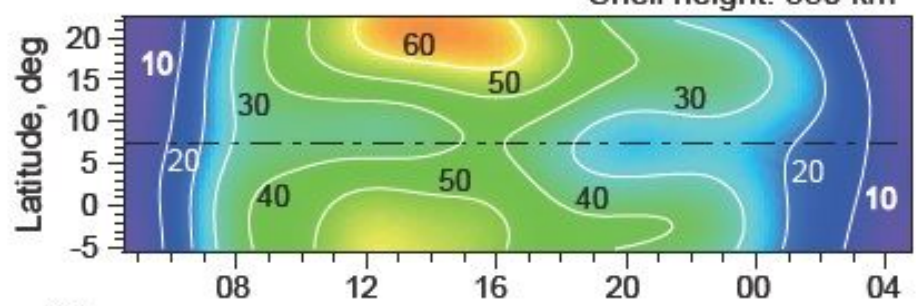

(c)

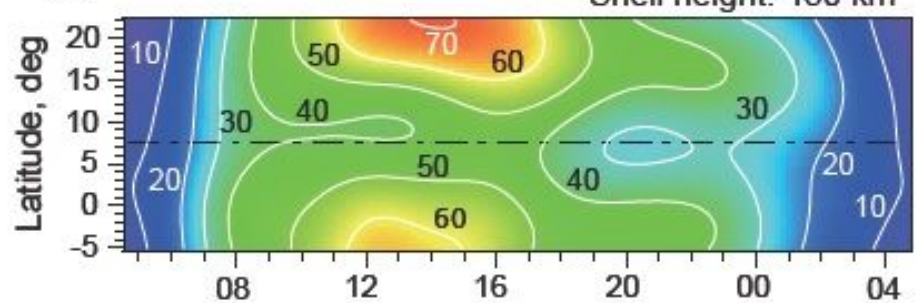

(d)

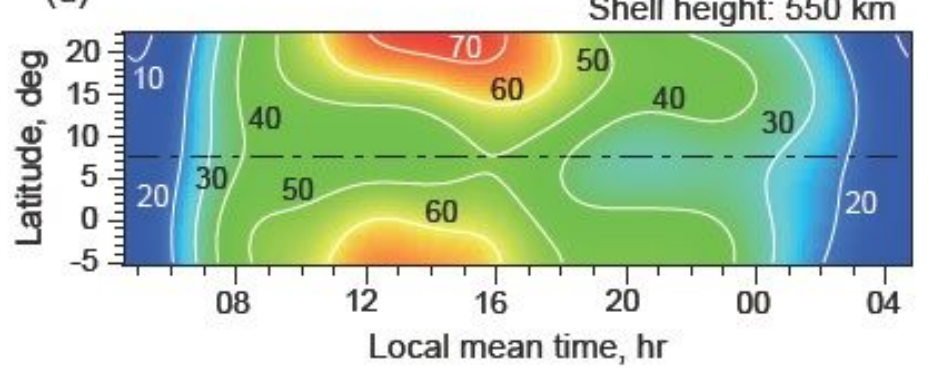

(e) $\left[\begin{array}{c}8 \text { TECu } \\ =4 \\ =0 \\ =-4 \\ -8\end{array}\right.$

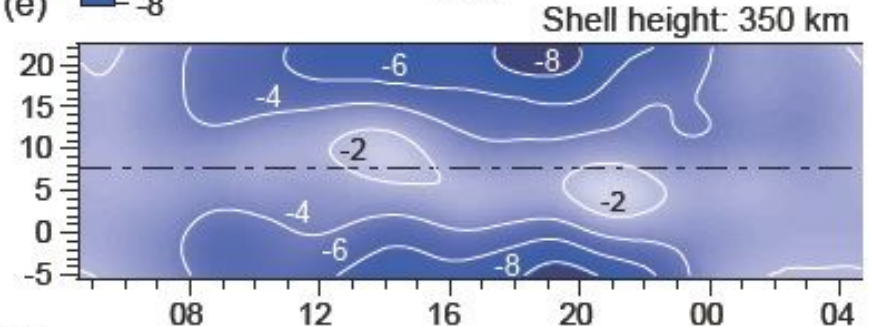

(f)

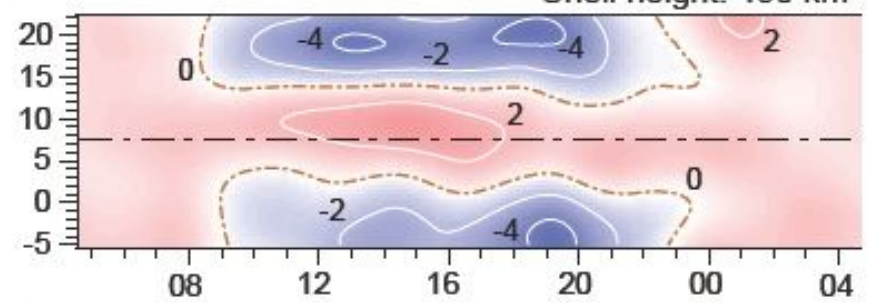

(g)

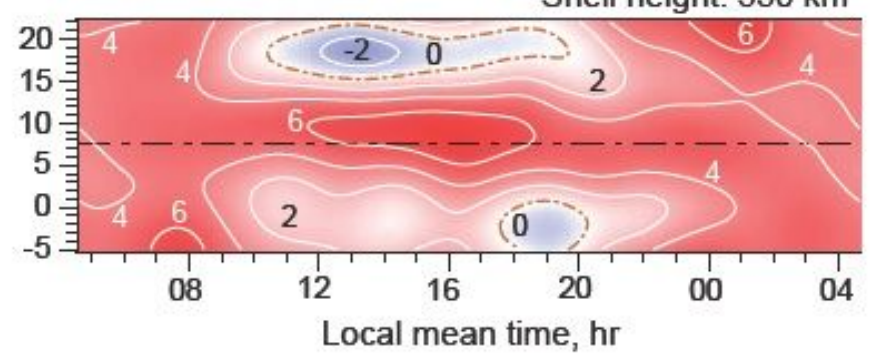

\section{Figure 5}

TEC maps. (a) Vertical TEC directly calculated from IRI model (IRI-direct TEC), (b) - (d) vertical TEC reconstructed by single-shell model for shell heights of 350,450 , and $550 \mathrm{~km}$, and (e) - (g) error of singleshell model reconstruction for each shell height. The dash-dotted line indicates the magnetic equator. 
Single-shell model (IRI simulation)

(a)

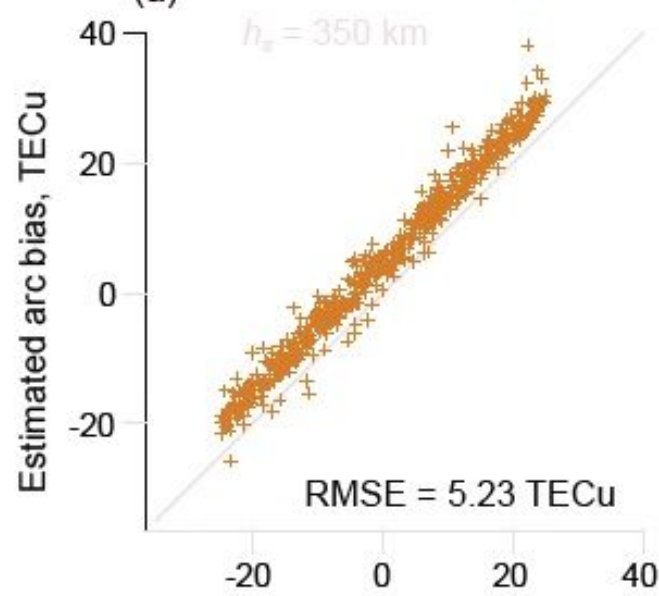

(b)

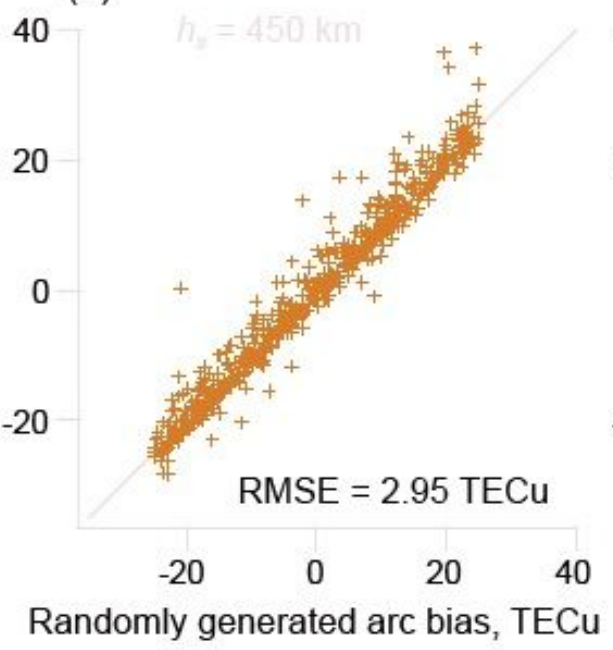

(c)

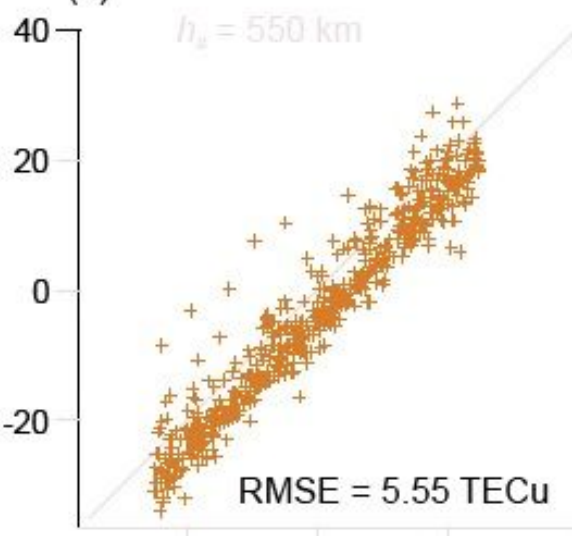

$\begin{array}{llll}-20 & 0 & 20 & 40\end{array}$

Double-shell model (IRI simulation)

(d)

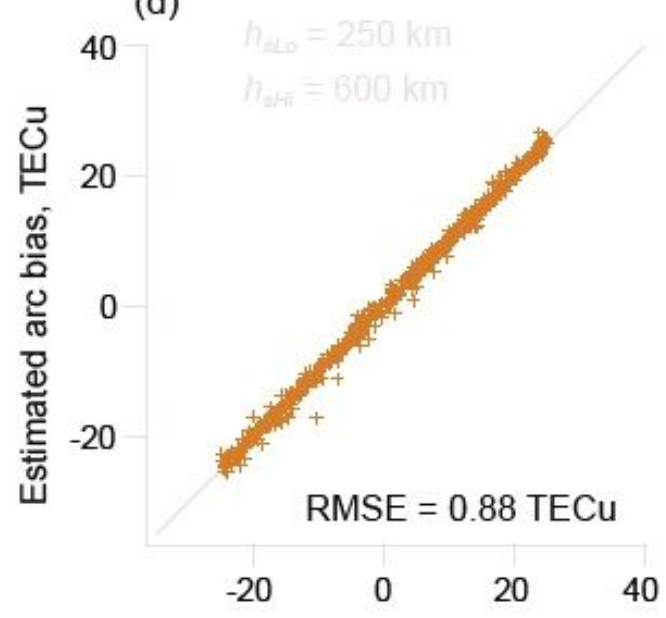

(e)

40

$-20$

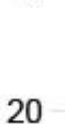

20

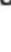

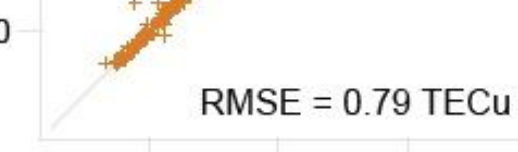

$\begin{array}{llll}-20 & 0 & 20 & 40\end{array}$

Randomly generated arc bias, TECu (f)

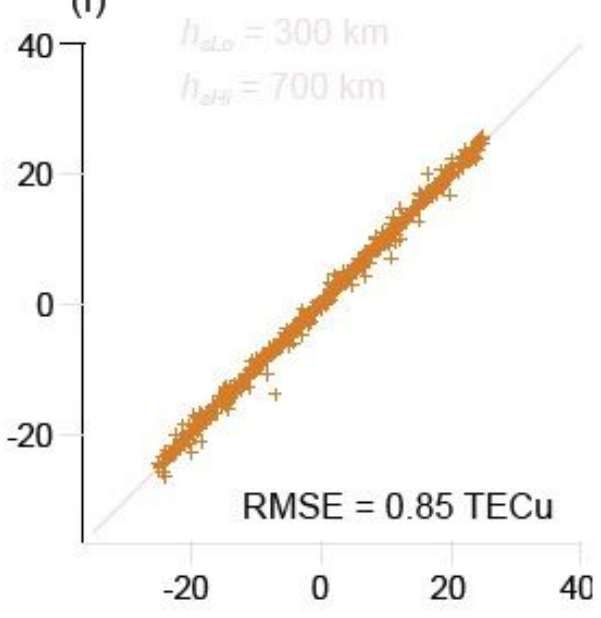

Figure 6

Estimation errors of arc biases. (a) - (c) Single-shell model with shell heights of 350, 450, and $550 \mathrm{~km}$. (d) - (f) Double-shell model with combinations of shell heights of 250/600, 300/600, and 300/700 km for lower/higher shells. 


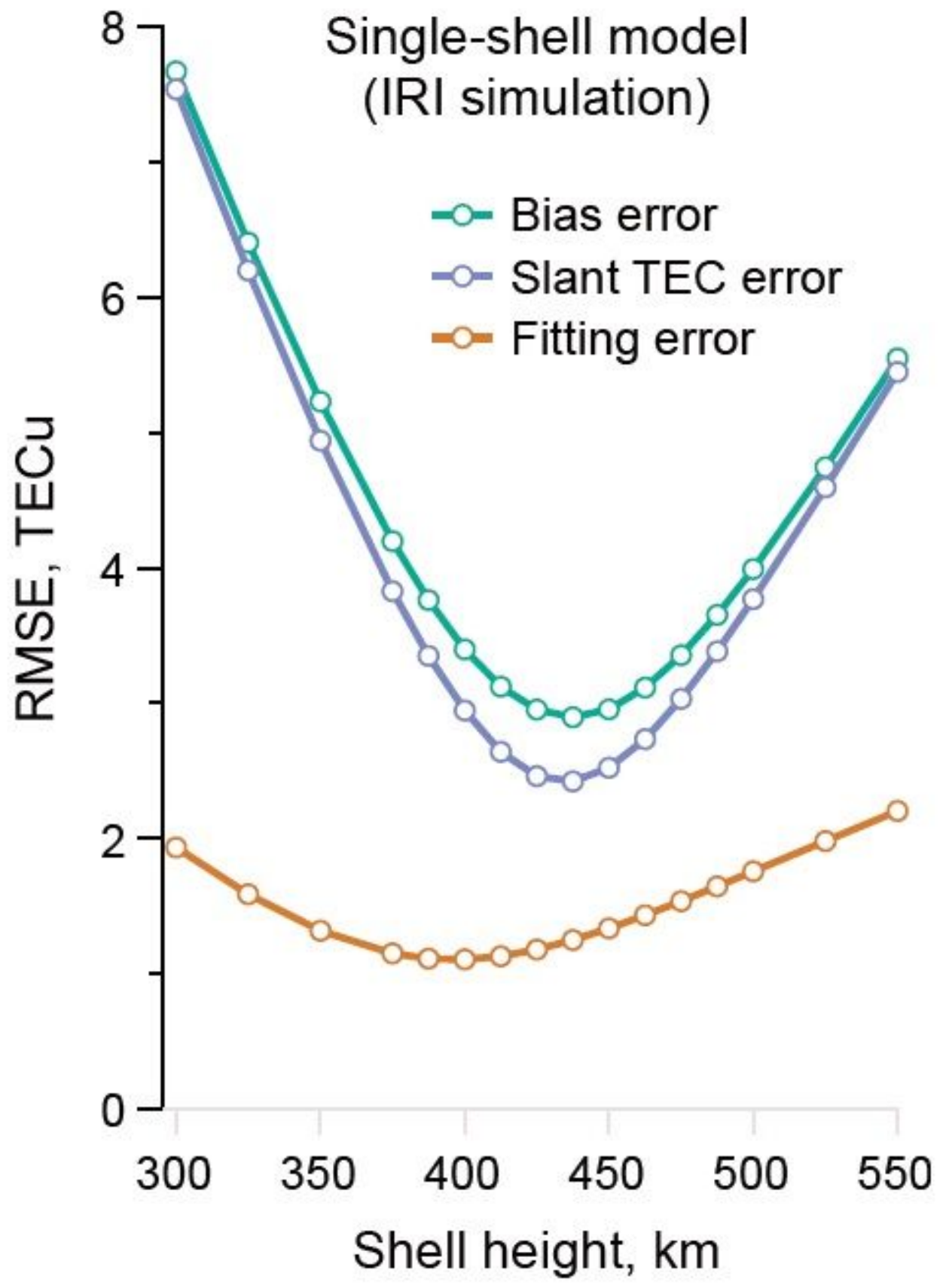

Figure 7

Functional fitting error, RMSE of bias estimation, and RMSE of slant TEC for different shell heights. 
Double-shell model (IRI simulation)
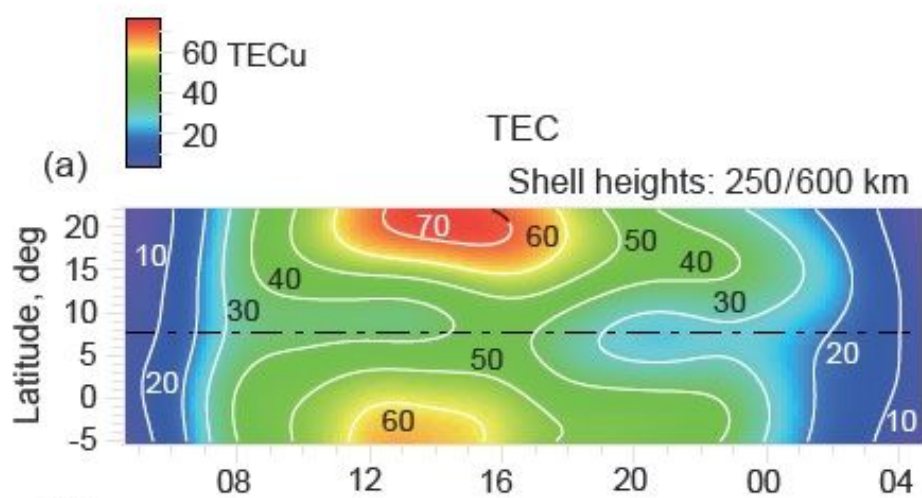

(b)

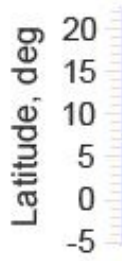

(c)

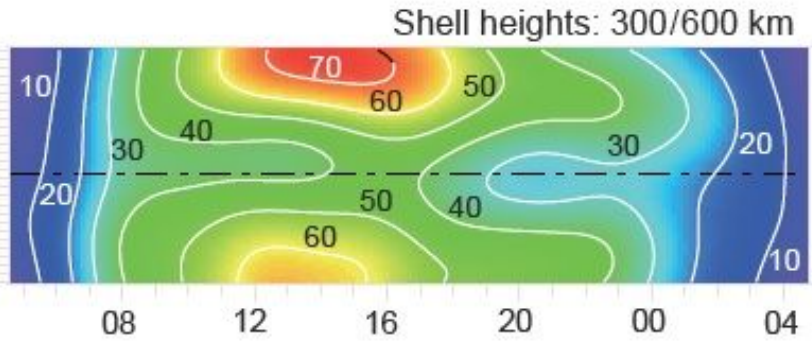

Shell heights: $300 / 700 \mathrm{~km}$

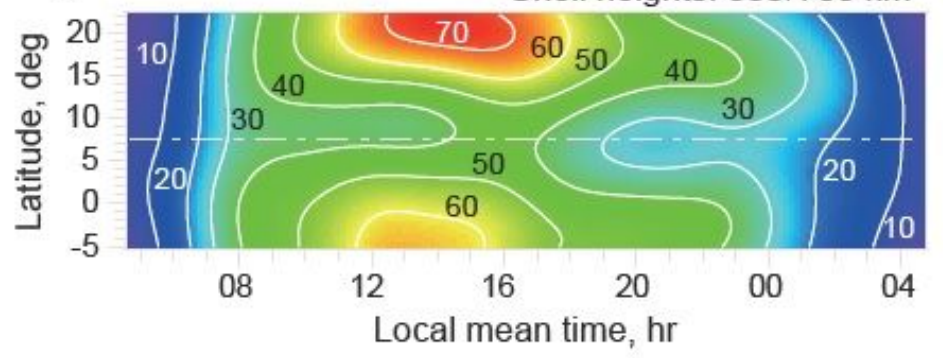

(d)

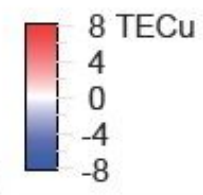

Error

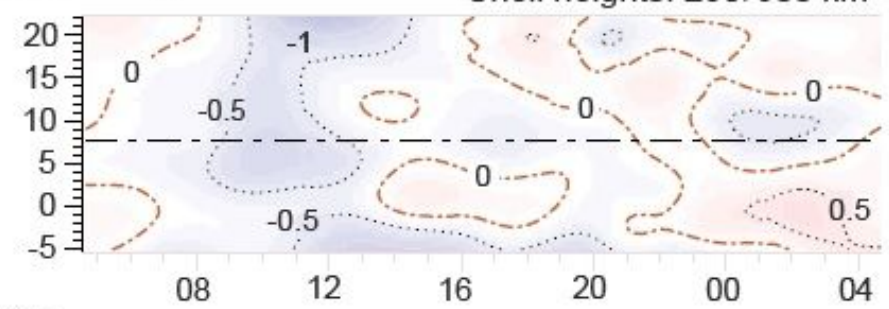

(e)

Shell heights: $300 / 600$ km

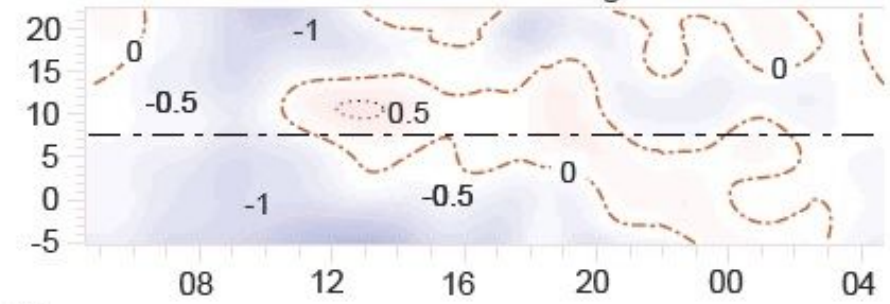

(f)

\section{Figure 8}

TEC maps. (a) - (c) Vertical TEC reconstructed by double-shell model for shell height combinations of 250/600, 300/600, and 300/700 km for lower/higher shells, and (d) - (f) TEC error for double-shell model reconstruction for each shell height combination. 


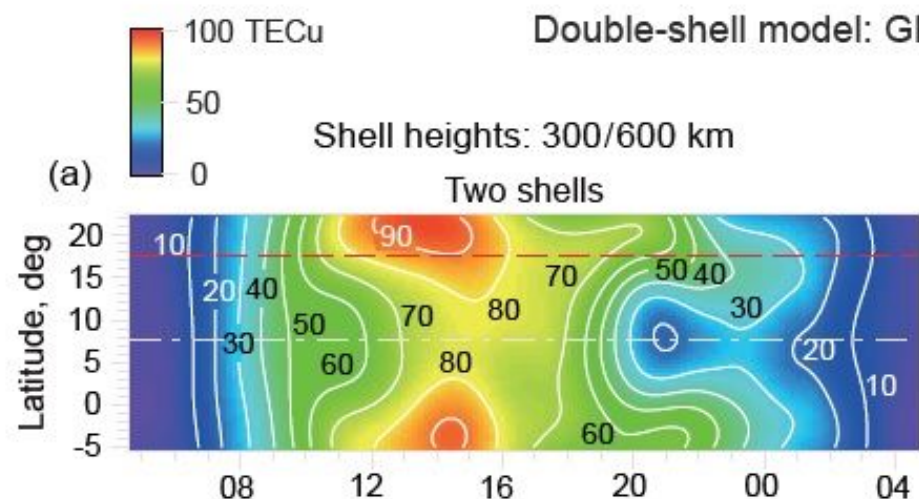

(b)

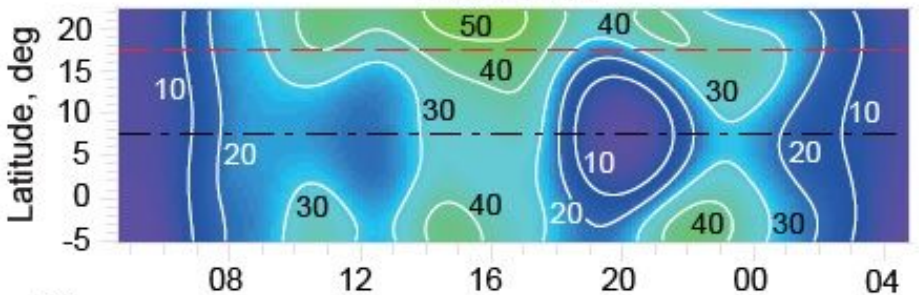

(c)

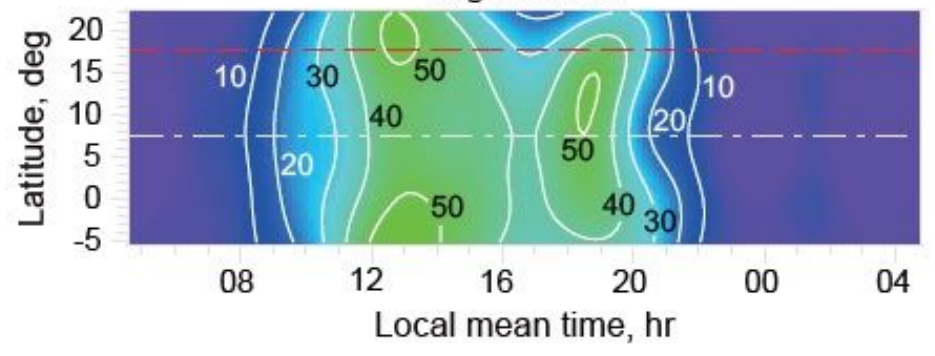

Shell heights: $280 / 700$ km

(d)

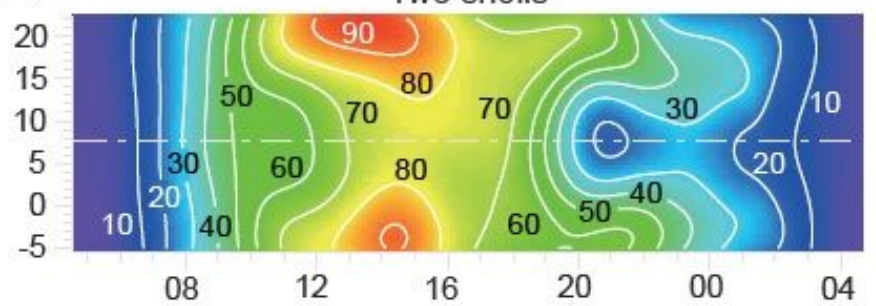

(e)

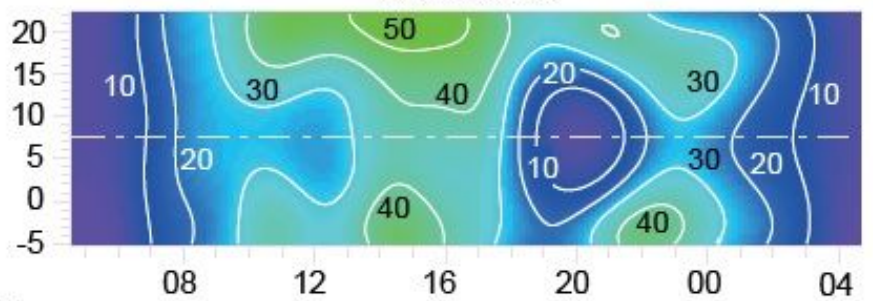

(f)

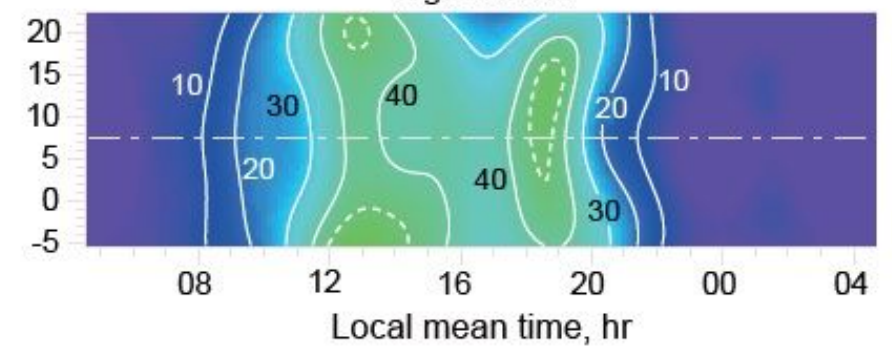

\section{Figure 9}

TEC maps derived from actual observations by double-shell model. (a) - (c) Vertical TEC, Is-TEC, and hsTEC for shell height combination of 300 and $600 \mathrm{~km}$. The red dashed line indicates a magnetic latitude of $12^{\circ} \mathrm{N}$. (d) - (f) Vertical TEC, Is-TEC, and hs-TEC for shell height combination of 280 and $700 \mathrm{~km}$. 
Single-shell model: GNSS, 16-17 March 2014

(a) 0

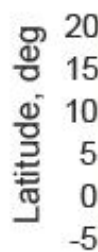

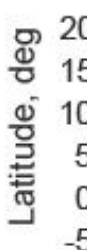

(b)

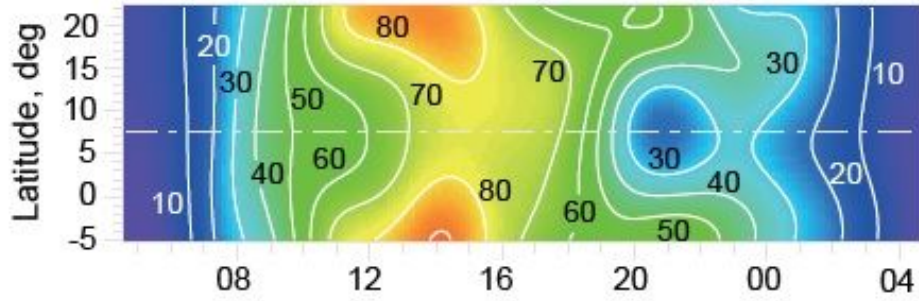

(c)

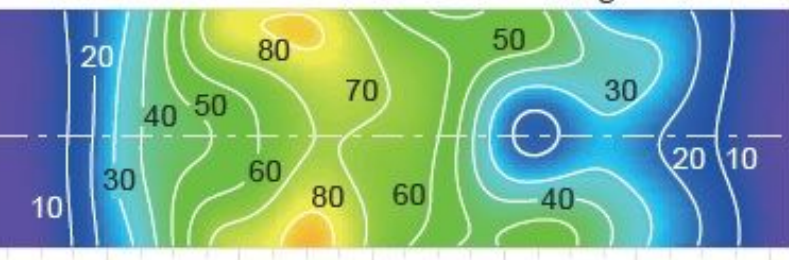

$08 \quad 12$

16

Shell height: $550 \mathrm{~km}$

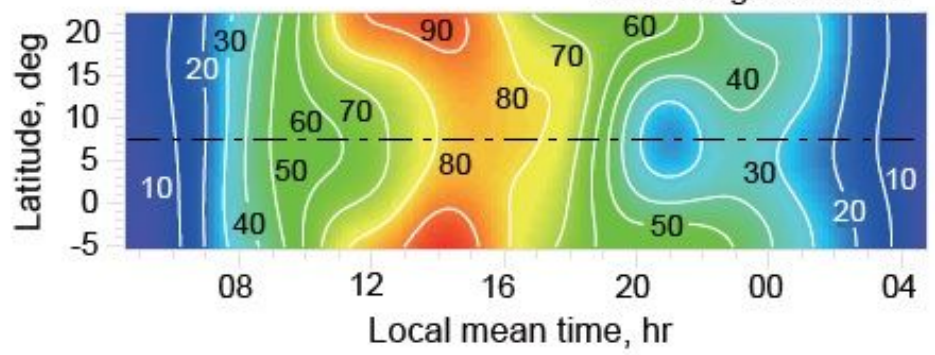

(d)
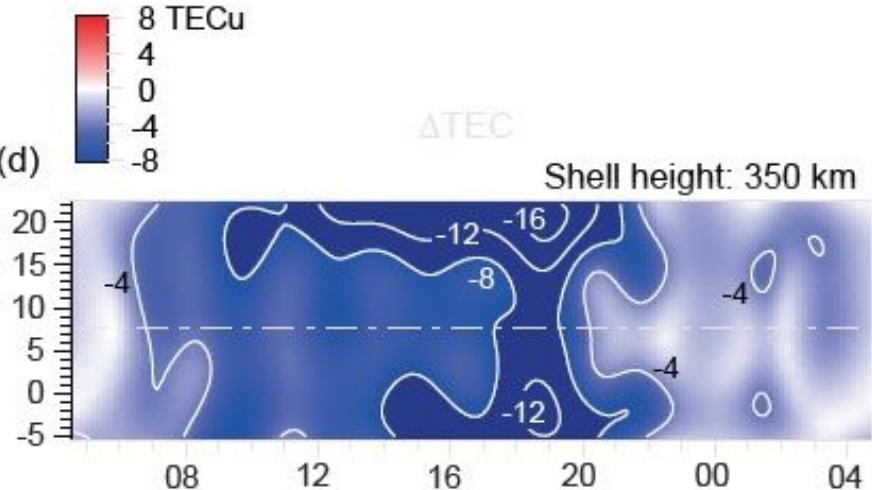

(e)

Shell height: $450 \mathrm{~km}$

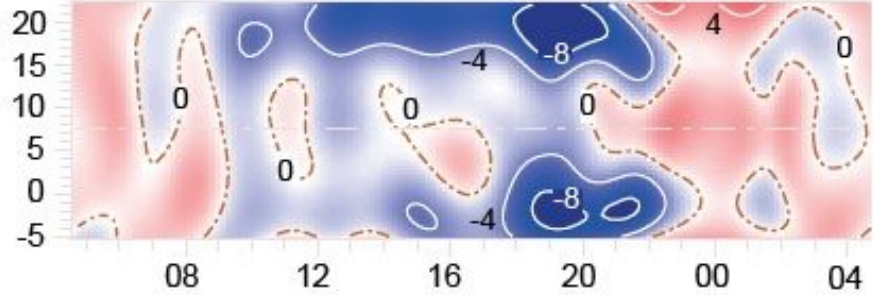

(f)

Shell height: $550 \mathrm{~km}$

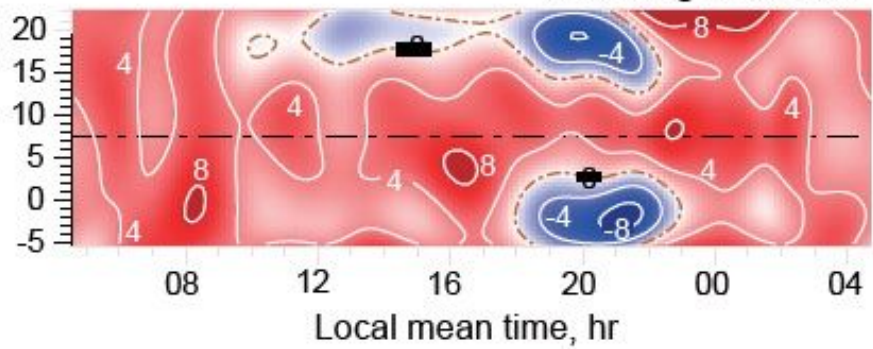

Figure 10

TEC maps derived from actual observations by single-shell model. (a) - (c) Vertical TEC for shell heights of 350,450 , and $550 \mathrm{~km}$, and (d) - (f) difference between left panels and double-shell TEC with shell height combination of 300 and $600 \mathrm{~km}$. 


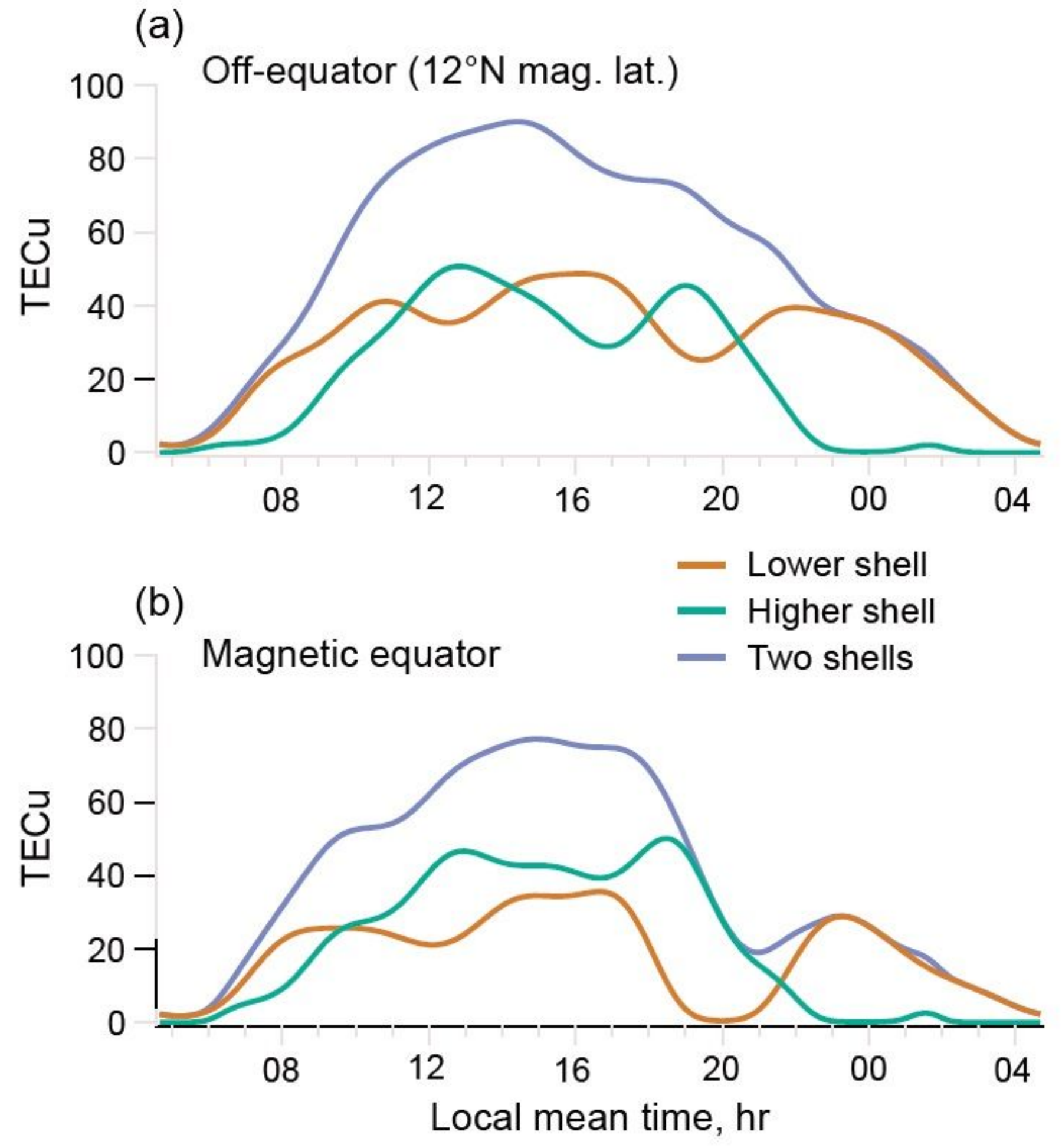

Figure 11

Local time variations of Is-TEC, hs-TEC, and two-shell TEC, at magnetic equator (a) and away from equator at $12^{\circ} \mathrm{N}$ magnetic latitude (b) with shell height combination of 300 and $600 \mathrm{~km}$. The two latitudes are shown by the dash-dotted line (magnetic equator) and red dashed line $\left(12^{\circ} \mathrm{N}\right.$ magnetic latitude) in Figures 9a-9c. 
(a)

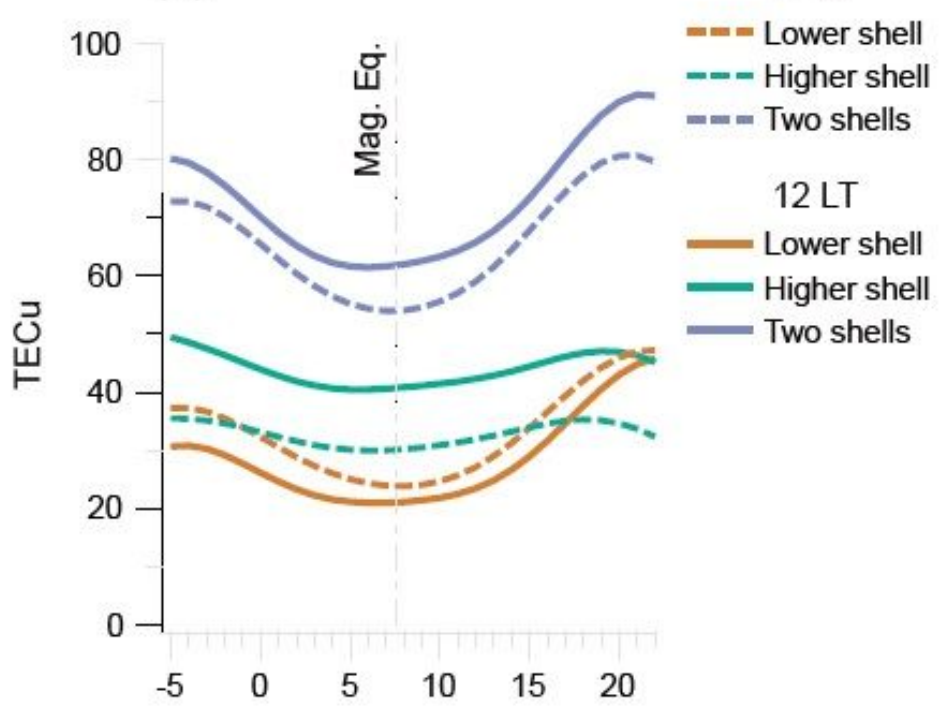

(c)

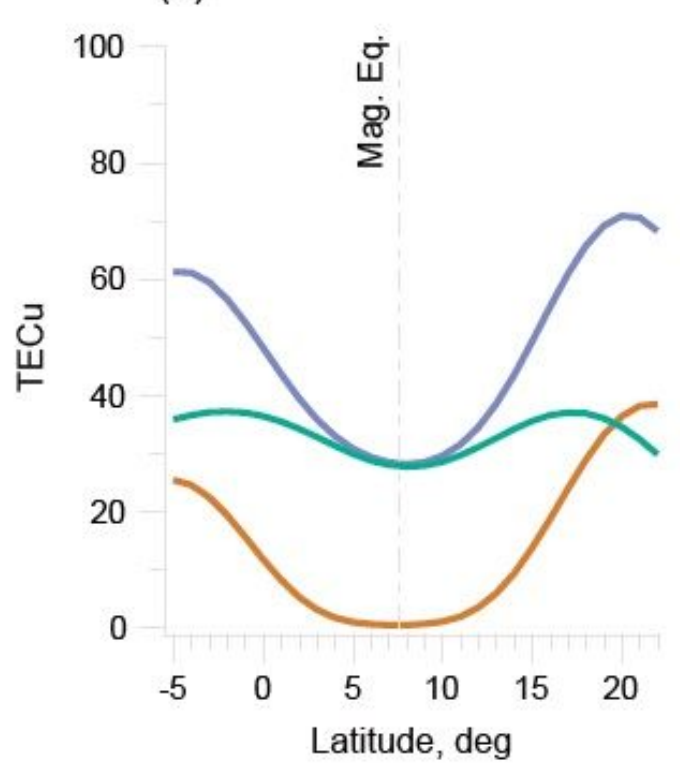

(b)

$17 \mathrm{LT}$

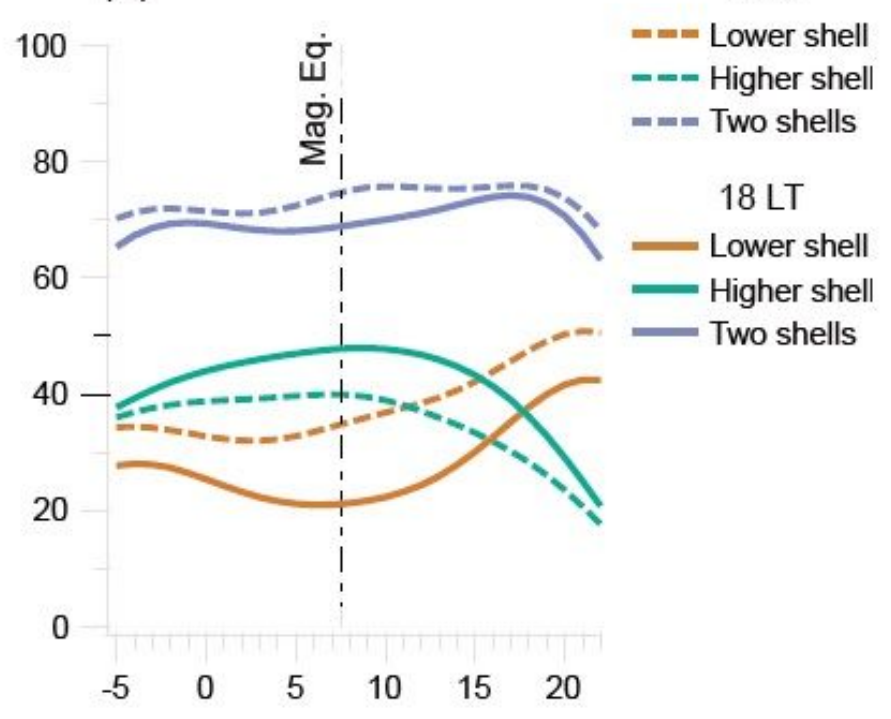

(d)

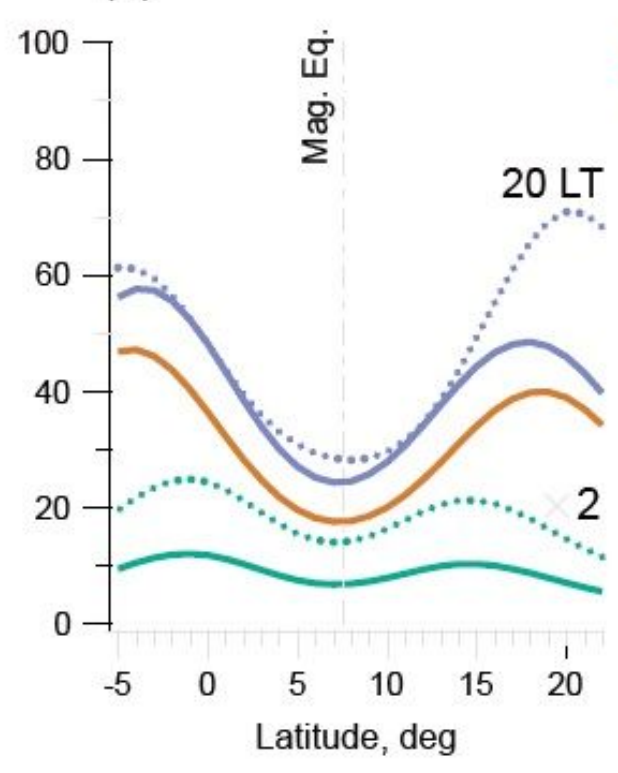

$22 \mathrm{LT}$

- Lower shell Higher shell Two shells

\section{Figure 12}

Latitudinal variation of IS-TEC, hs-TEC, and two-shell TEC with shell height combination of 300 and 600 km. (a) Transition from 11 to 12 LT and (b) transition from 17 to $18 \mathrm{LT}$, (c) $20 \mathrm{LT}$, and (d) 22 LT with plots of two-shell TEC for 20 LT (purple dotted line) and doubled hs-TEC (green dotted line) for convenience of comparison.

\section{Supplementary Files}

This is a list of supplementary files associated with this preprint. Click to download. 
- graphicalabstract.png 